\title{
Prediction of Physical-Chemical
}

\section{and Fire Hazard Characteristics by Carbon Chain Rules.}

\section{Carboxylic Acids}

\author{
Sergey G. Alexeev*a,b, Kirill S. Alexeev ${ }^{a, c}$, \\ Nicolay M. Barbin ${ }^{\mathrm{b}, \mathrm{c}}$ and Dar'ya L. Alexeeva ${ }^{\mathrm{d}}$ \\ ${ }^{a}$ Science and Engineering Centre \\ "Reliability and Safety of Large Systems and Mashines" UB RAS \\ 54a Studencheskaya Str., Yekaterinburg, 620049, Russia \\ ${ }^{b}$ Ural Institute of State Fire Service of Emercom of Russia \\ 22 Mira Str., Yekaterinburg, 620062, Russia \\ ${ }^{c}$ Ural State Agrarian University \\ 42 Karla Libknekhta Str., Yekaterinburg, 620075, Russia \\ ${ }^{d}$ Chemical Technology Institute of Ural Federal University \\ named by the first President of Russia B.N. El'tzin \\ 28 Mira Str., Yekaterinburg, 620062, Russia
}

Received 01.08.2018, received in revised form 12.11.2018, accepted 05.02.2019

Investigation of the dependence of physico-chemical and fire hazard properties from the chemical structure of carboxylic acids is carried out. Forecasting of the boiling temperature, the flash point, the temperature and the concentration flammability limits, the heats of combustion and vaporization is performed by the carbon chain rules (CCR). The following empirical equations for the calculation of physico-chemical and fire hazard indices from the conventional carbon chain and from the number of carbon atoms are proposed for the convenience of practical application of the CCR. A comparative analysis of the proposed methods for the flash point calculating and the already known methods of GOST 12.1.044-89, Mendeleev and ACD/Lab 2014 is carried out. It is shown, basically, that the new methods give more accurate calculation results than the comparison design procedures.

Keywords: boiling point, flash point, temperature flammability limits, flammability limits, heat of combustion, heat of vaporization, carboxylic acid.

Citation: Alexeev S.G., Alexeev K.S., Barbin N.M., Alexeeva D.L. Prediction of physical-chemical and fire hazard characteristics by carbon chain rules. 2. Carboxylic acids, J. Sib. Fed. Univ. Chem., 2019, 12(1), 18-30. DOI: 10.17516/1998-2836-0105.

(C) Siberian Federal University. All rights reserved

* Corresponding author E-mail address: 3608113@mail.ru 


\title{
Прогнозирования физико-химических и пожаропасных показателей с помощью правил углеродной цепи.
}

\section{2. Карбоновые кислоты}

\author{
С.Г. Алексеев ${ }^{\mathrm{a}, \boldsymbol{\sigma}}$, К.С. Алексеев ${ }^{\mathrm{a}, \mathrm{s}}$, \\ Н.М. Барбинб,в, Д.Л. Алексеева ${ }^{\text {г }}$ \\ ${ }^{a}$ НИЦ «Надежность и ресурс больших систем и машин» УрО РАН \\ Россия, 620049, Екатеринбург, ул. Студенческая, $54 a$ \\ ${ }^{\circ}$ Уральский институт ГПС МЧС России \\ Россия, 620062, Екатеринбург, ул. Мира, 22 \\ ${ }^{8}$ Уральский государственный аграрный университет \\ Россия, 620075, Екатеринбург, ул. Карла Либкнехта, 42 \\ 'Химико-технологический институт \\ Уральского федерального университета \\ имени первого Президента России Б.Н. Ельцина \\ Россия, 620062, Екатеринбург, ул. Мира, 28
}

Проведено исследование зависимости физико-химических $и$ пожароопасных свойств от химического строения карбоновых кислот. С помощью правил углеродной иепи выполнено прогнозирование температур кипения и вспышки, температурных и кониентрационных пределов воспламенения, теплот сгорания и парообразования. Для удобства практического применения правил углеродной цепи предложены следующие эмпирические уравнения для расчета физико-химических и пожароопасных показателей от условной углеродной цепи и от числа атомов углерода. Проведен сравнительный анализ предлагаемых методов расчета температуры вспыики с методами ГОСТ 12.1.044-89, Менделеева и ACD/Lab 2014. Показано, что новые методы в основном дают более точные результаты расчета, чем методы сравнения.

Ключевые слова: температура кипения, температура вспышки, температурные предель воспламенения, концентрационные предель воспламенения, теплота сгорания, теплота парообразования, карбоновая кислота.

\section{Introduction}

In the first part of this series of works it is noted [1] that the rate of accumulation of data on physico-chemical, fire hazard and other properties of organic compounds lag far behind either the process of their production by organic synthesis or extraction from natural raw materials and industrial wastes. In that regard, the calculation methods should reduce at least this imbalance. As part of the solution to this problem, a new direction in chemistry, which was called chemoinformatics, was appeared. This term shall be construed to mean "the use of informatics methods to solve chemical problems" [2]. 


\section{Objects, methods and results}

The 77 representatives of the class of aliphatic carboxylic acids were selected as objects of study (Table 1). Data for the normal boiling point $(N B P)$, the flash point $(F P)$, the temperature flammability limits $(L F T L, U F T L)$, the flammability limits $(L F L, U F L)$, the heat of combustion $\left(H_{\text {comb }}\right)$ and the heat of vaporization $\left(H_{v a p}\right)$ of these carboxylic acids is taken from the Handbook of Yaws and DIPPR 801 database $[3,4]$. The Table 1 provides the experimental data highlighted in normal font, prediction of physico-chemical and fire hazard properties - in italic, and the abnormal values that were not considered in the present work - in bold type.

Table 1. Reference and calculated data of physicochemical and fire hazard properties of carboxylic acids

\begin{tabular}{|c|c|c|c|c|c|c|c|c|}
\hline \multirow{2}{*}{$\begin{array}{c}\text { Acid, } \\
\text { number }(\mathrm{CCC})\end{array}$} & $N B P$ & $F P$ & $L F T L$ & $U F T L$ & $L F L$ & $U F L$ & $-H_{\text {comb }}$ & $H_{\text {vap }}$ \\
\hline & \multicolumn{4}{|c|}{ K } & \multicolumn{2}{|c|}{$\%$ (vol.) } & \multicolumn{2}{|c|}{$\mathrm{kJ} / \mathrm{mol}$} \\
\hline 1 & 2 & 3 & 5 & 6 & 7 & 8 & 9 & 10 \\
\hline $\begin{array}{l}\text { Formic } \\
1(1.0)\end{array}$ & $\begin{array}{l}373.9^{1} \\
368.0^{3} \\
371.3^{4} \\
373.7^{5}\end{array}$ & $\begin{array}{l}323.2^{2} \\
294.1^{3} \\
292.8^{4} \\
303.0^{5} \\
300.9^{6} \\
305.5^{7}\end{array}$ & $\begin{array}{l}314.9^{2} \\
294.1^{3} \\
294.6^{4} \\
302.1^{8}\end{array}$ & $\begin{array}{l}344.3^{2} \\
333.7^{3} \\
325.5^{4} \\
330.1^{9}\end{array}$ & $12.0^{2}$ & $50.0^{2}$ & $\begin{array}{l}211.5^{2} \\
178.2^{3} \\
189.3^{4}\end{array}$ & $\begin{array}{l}32.10^{1} \\
31.60^{3} \\
31.89^{4} \\
22.69^{5}\end{array}$ \\
\hline $\begin{array}{l}\text { Acetic } \\
2(2.0)\end{array}$ & $\begin{array}{l}391.0^{1} \\
394.0^{3} \\
394.0^{4} \\
390.2^{5}\end{array}$ & $\begin{array}{l}312.1^{2} \\
315.0^{3} \\
311.6^{4} \\
313.2^{5} \\
313.0^{6} \\
317.0^{7}\end{array}$ & $\begin{array}{l}310.6^{2} \\
315.0^{3} \\
311.3^{4} \\
312.4^{8}\end{array}$ & $\begin{array}{l}345.6^{2} \\
341.6^{3} \\
343.0^{4} \\
342.4^{9}\end{array}$ & $\begin{array}{l}4.0^{2} \\
3.8^{3} \\
4.0^{4} \\
4.6^{10}\end{array}$ & $\begin{array}{l}19.9^{2} \\
14.0^{3} \\
19.3^{4} \\
22.3^{I I}\end{array}$ & $\begin{array}{l}786.6^{2} \\
782.0^{3} \\
796.9^{4} \\
882.6^{12}\end{array}$ & $\begin{array}{l}33.73^{1} \\
33.80^{3} \\
34.02^{4} \\
23.70^{5}\end{array}$ \\
\hline $\begin{array}{l}\text { Propionic } \\
\mathbf{3}(3.0)\end{array}$ & $\begin{array}{l}414.0^{1} \\
413.5^{3} \\
415.8^{4} \\
414.8^{5}\end{array}$ & $\begin{array}{l}330.1^{2} \\
328.7^{3} \\
329.3^{4} \\
324.8^{5} \\
329.3^{6} \\
332.3^{7}\end{array}$ & $\begin{array}{l}328.1^{2} \\
328.7^{3} \\
327.2^{4} \\
326.5^{8}\end{array}$ & $\begin{array}{l}357.5^{2} \\
359.5^{3} \\
359.8^{4} \\
359.0^{9}\end{array}$ & $\begin{array}{l}2.9^{2} \\
3.0^{3} \\
2.9^{4} \\
2.9^{10}\end{array}$ & $\begin{array}{l}12.0^{2} \\
12.2^{3} \\
11.6^{4} \\
15.6^{11}\end{array}$ & $\begin{array}{l}1395.0^{2} \\
1397.3^{3} \\
1404.4^{4} \\
1498.1^{5}\end{array}$ & $\begin{array}{l}35.89^{1} \\
35.90^{3} \\
36.08^{4} \\
40.08^{5}\end{array}$ \\
\hline $\begin{array}{l}\text { Butyric } \\
4(4.0)\end{array}$ & $\begin{array}{l}436.0^{1} \\
436.5^{3} \\
436.7^{4} \\
437.5^{5}\end{array}$ & $\begin{array}{l}345.2^{2} \\
344.7^{3} \\
345.7^{4} \\
349.8^{5} \\
344.9^{6} \\
347.0^{7}\end{array}$ & $\begin{array}{l}340.6^{2} \\
344.7^{3} \\
342.2^{4} \\
340.0^{8}\end{array}$ & $\begin{array}{l}373.4^{2} \\
372.8^{3} \\
375.8^{4} \\
374.9^{9}\end{array}$ & $\begin{array}{l}2.0^{2} \\
2.3^{3} \\
2.0^{4} \\
2.1^{10}\end{array}$ & $\begin{array}{c}10.0^{2} \\
9.9^{3} \\
9.8^{4} \\
11.9^{11}\end{array}$ & $\begin{array}{l}2008.0^{2} \\
2006.0^{3} \\
2012.0^{4} \\
2113.7^{12}\end{array}$ & $\begin{array}{l}37.99^{1} \\
38.00^{3} \\
38.04^{4} \\
42.39^{5}\end{array}$ \\
\hline $\begin{array}{l}\text { Valeric } \\
\mathbf{5}(5.0)\end{array}$ & $\begin{array}{l}459.0^{1} \\
456.5^{3} \\
456.7^{4} \\
458.4^{5}\end{array}$ & $\begin{array}{c}359.2^{2} \\
360.2^{3} \\
361.1^{4} \\
362.0^{5} \\
361.2^{6} \\
362.3^{7}\end{array}$ & $\begin{array}{l}357.1^{2} \\
360.2^{3} \\
356.3^{4} \\
354.1^{5}\end{array}$ & $\begin{array}{l}388.1^{2} \\
392.4^{3} \\
391.0^{4} \\
391.5^{9}\end{array}$ & $\begin{array}{l}1.6^{2} \\
1.7^{3} \\
1.6^{4} \\
1.7^{10}\end{array}$ & $\begin{array}{l}7.8^{2} \\
8.0^{3} \\
8.5^{4} \\
9.7^{11}\end{array}$ & $\begin{array}{l}2617.0^{2} \\
2619.0^{3} \\
2619.5^{4} \\
2729.2^{12}\end{array}$ & $\begin{array}{l}40.19^{1} \\
40.00^{3} \\
39.92^{4} \\
44.57^{5}\end{array}$ \\
\hline $\begin{array}{l}\text { Caproic } \\
6(6.0)\end{array}$ & $\begin{array}{l}477.0^{1} \\
477.0^{3} \\
475.8^{4} \\
477.8^{5}\end{array}$ & $\begin{array}{l}375.2^{2} \\
373.7^{3} \\
375.3^{4} \\
377.6^{5} \\
373.9^{6} \\
374.4^{7}\end{array}$ & $\begin{array}{l}371.1^{2} \\
373.7^{3} \\
369.5^{4} \\
365.0^{8}\end{array}$ & $\begin{array}{l}411.4^{2} \\
404.1^{3} \\
405.4^{4} \\
404.5^{9}\end{array}$ & $\begin{array}{l}1.3^{2} \\
1.4^{3} \\
1.3^{4} \\
1.4^{10}\end{array}$ & $\begin{array}{l}9.3^{\mathbf{2}} \\
7.5^{3} \\
7.6^{4} \\
8.2^{11}\end{array}$ & $\begin{array}{l}3230.0^{2} \\
3228.0^{3} \\
3227.0^{4} \\
3344.7^{12}\end{array}$ & $\begin{array}{l}41.92^{1} \\
41.90^{3} \\
41.72^{4} \\
46.61^{5}\end{array}$ \\
\hline $\begin{array}{l}\text { Enanthic } \\
7(7.0)\end{array}$ & $\begin{array}{l}495.0^{1} \\
493.5^{3} \\
494.0^{4} \\
495.7^{5}\end{array}$ & $\begin{array}{l}388.2^{2} \\
387.7^{3} \\
388.4^{4} \\
372.4^{5} \\
386.6^{6} \\
386.4^{7}\end{array}$ & $\begin{array}{l}381.9^{2} \\
387.7^{3} \\
381.9^{4} \\
375.9^{8}\end{array}$ & $\begin{array}{l}420.1^{2} \\
421.5^{3} \\
419.1^{4} \\
417.5^{9}\end{array}$ & $\begin{array}{l}1.1^{2} \\
1.2^{3} \\
1.1^{4} \\
1.2^{10}\end{array}$ & $\begin{array}{l}7.2^{2} \\
6.9^{3} \\
6.9^{4} \\
7.2^{11}\end{array}$ & $\begin{array}{l}3839.0^{2} \\
3839.0^{3} \\
3834.6^{4} \\
3960.2^{12}\end{array}$ & $\begin{array}{l}43.65^{1} \\
43.50^{3} \\
43.43^{4} \\
48.52^{5}\end{array}$ \\
\hline
\end{tabular}


Continued Table 1

\begin{tabular}{|c|c|c|c|c|c|c|c|c|}
\hline 1 & 2 & 3 & 5 & 6 & 7 & 8 & 9 & 10 \\
\hline $\begin{array}{l}\text { Caproic } \\
\mathbf{8}(8.0)\end{array}$ & $\begin{array}{l}510.0^{1} \\
511.1^{3} \\
511.4^{4} \\
512.5^{5}\end{array}$ & $\begin{array}{l}400.2^{2} \\
400.7^{3} \\
400.3^{4} \\
380.5^{5} \\
397.3^{6} \\
396.5^{7}\end{array}$ & $\begin{array}{l}392.9^{2} \\
400.7^{3} \\
393.4^{4} \\
384.8^{8}\end{array}$ & $\begin{array}{l}431.6^{2} \\
431.9^{3} \\
432.0^{4} \\
428.3^{9}\end{array}$ & $\begin{array}{l}1.0^{2} \\
1.0^{3} \\
1.0^{4} \\
1.0^{10}\end{array}$ & $\begin{array}{l}6.4^{2} \\
6.6^{3} \\
6.4^{4} \\
6.7^{11}\end{array}$ & $\begin{array}{l}4448.0^{2} \\
4450.0^{3} \\
4442.1^{4} \\
4575.7^{12}\end{array}$ & $\begin{array}{l}45.10^{1} \\
45.20^{3} \\
45.06^{4} \\
50.32^{5}\end{array}$ \\
\hline $\begin{array}{l}\text { Pelargonic } \\
\mathbf{9}(9.0)\end{array}$ & $\begin{array}{l}527.2^{1} \\
525.0^{3} \\
527.8^{4} \\
528.1^{5}\end{array}$ & $\begin{array}{l}413.2^{2} \\
410.7^{3} \\
411.1^{4} \\
373.2^{5} \\
409.4^{6} \\
408.0^{7}\end{array}$ & $\begin{array}{l}404.1^{2} \\
410.7^{3} \\
404.0^{4} \\
395.1^{8}\end{array}$ & $\begin{array}{l}443.6^{2} \\
443.1^{3} \\
444.1^{4} \\
440.8^{9}\end{array}$ & $\begin{array}{l}0.9^{2} \\
0.9^{3} \\
0.9^{4} \\
0.9^{10}\end{array}$ & $\begin{array}{l}5.9^{2} \\
6.0^{3} \\
6.0^{4} \\
6.2^{11}\end{array}$ & $\begin{array}{l}5061.0^{2} \\
5084.0^{3} \\
5049.7^{4} \\
5191.3^{12}\end{array}$ & $\begin{array}{l}46.76^{1} \\
46.10^{3} \\
46.61^{4} \\
52.03^{5}\end{array}$ \\
\hline $\begin{array}{l}\text { Caproic } \\
\mathbf{1 0}(10.0)\end{array}$ & $\begin{array}{l}\mathbf{5 3 0 . 0}^{1} \\
543.4^{3} \\
543.3^{4} \\
542.7^{5}\end{array}$ & $\begin{array}{l}421.2^{2} \\
420.7^{3} \\
420.7^{4} \\
394.9^{5}\end{array}$ & $\begin{array}{l}413.1^{2} \\
420.7^{3} \\
413.8^{4}\end{array}$ & $\begin{array}{l}454.6^{2} \\
454.1^{3} \\
455.4^{4}\end{array}$ & $\begin{array}{l}0.8^{2} \\
0.8^{3} \\
0.8^{4} \\
0.8^{10}\end{array}$ & $\begin{array}{l}5.5^{2} \\
5.6^{3} \\
5.6^{4} \\
5.8^{11}\end{array}$ & $\begin{array}{c}5720.0^{2} \\
5657.0^{3} \\
5657.2^{4} \\
5806.8^{12}\end{array}$ & $\begin{array}{l}47.04^{1} \\
48.20^{3} \\
48.07^{4} \\
53.63^{5}\end{array}$ \\
\hline $\begin{array}{l}\text { Undecylic } \\
\mathbf{1 1}(11.0)\end{array}$ & $\begin{array}{l}557.2^{1} \\
556.7^{3} \\
557.9^{4} \\
556.4^{5}\end{array}$ & $\begin{array}{l}428.1^{2} \\
429.2^{3} \\
429.2^{4} \\
401.3^{5} \\
430.7^{6} \\
428.1^{7}\end{array}$ & $\begin{array}{l}421.7^{2} \\
429.2^{3} \\
422.6^{4} \\
412.9^{8}\end{array}$ & $\begin{array}{l}464.6^{2} \\
465.6^{3} \\
466.0^{4} \\
462.4^{9}\end{array}$ & $\begin{array}{l}0.7^{2} \\
0.7^{3} \\
0.7^{4} \\
0.7^{10}\end{array}$ & $\begin{array}{l}5.2^{2} \\
5.2^{3} \\
5.3^{4} \\
5.4^{11}\end{array}$ & $\begin{array}{l}6253.0^{2} \\
6285.0^{3} \\
6264.7^{4} \\
6422.3^{12}\end{array}$ & $\begin{array}{l}49.68^{1} \\
49.00^{3} \\
49.44^{4} \\
55.15^{5}\end{array}$ \\
\hline $\begin{array}{l}\text { Lauric } \\
12(12.0)\end{array}$ & $\begin{array}{l}571.0^{1} \\
571.3^{3} \\
571.7^{4} \\
569.3^{5}\end{array}$ & $\begin{array}{l}437.1^{2} \\
435.1^{3} \\
436.5^{4} \\
407.3^{5} \\
440.5^{6} \\
437.4^{7}\end{array}$ & $\begin{array}{l}429.7^{2} \\
435.1^{3} \\
430.6^{4} \\
421.0^{8}\end{array}$ & $\begin{array}{l}476.6^{2} \\
474.9^{3} \\
475.8^{4} \\
472.4^{9}\end{array}$ & $\begin{array}{l}0.6^{2} \\
0.7^{3} \\
0.6^{4} \\
0.7^{10}\end{array}$ & $\begin{array}{l}5.1^{2} \\
5.1^{3} \\
5.0^{4} \\
5.1^{11}\end{array}$ & $\begin{array}{l}6850.0^{2} \\
6853.0^{3} \\
6872.3^{4} \\
7037.8^{5}\end{array}$ & $\begin{array}{l}51.03^{1} \\
50.80^{3} \\
50.73^{4} \\
56.59^{5}\end{array}$ \\
\hline $\begin{array}{l}\text { Tridecylic } \\
13(13.0)\end{array}$ & $\begin{array}{l}580.6^{1} \\
585.3^{2} \\
584.8^{3} \\
584.5^{4} \\
581.4^{5}\end{array}$ & $\begin{array}{l}442.1^{2} \\
446.1^{3} \\
442.7^{4} \\
412.8^{5} \\
450.6^{6} \\
447.0^{7}\end{array}$ & $\begin{array}{l}439.1^{2} \\
446.1^{3} \\
437.7^{4} \\
429.4^{8}\end{array}$ & $\begin{array}{l}485.1^{2} \\
488.6^{3} \\
484.8^{4} \\
482.7^{9}\end{array}$ & $\begin{array}{l}0.6^{2} \\
0.5^{3} \\
0.6^{4} \\
0.6^{10}\end{array}$ & $\begin{array}{l}4.9^{2} \\
5.0^{3} \\
4.8^{4} \\
4.8^{I I}\end{array}$ & $\begin{array}{l}7453.0^{2} \\
7447.0^{3} \\
7479.8^{4} \\
7653.4^{12}\end{array}$ & $\begin{array}{l}51.97^{1} \\
52.40^{3} \\
51.94^{4} \\
57.96^{5}\end{array}$ \\
\hline $\begin{array}{l}\text { Isobutanoic } \\
\mathbf{1 4}(3.5)\end{array}$ & $\begin{array}{l}427.0^{1} \\
425.0^{3} \\
426.4^{4} \\
428.4^{5}\end{array}$ & $\begin{array}{l}333.2^{2} \\
337.7^{3} \\
337.6^{4} \\
328.4^{5} \\
338.5^{6} \\
341.1^{7}\end{array}$ & $\begin{array}{l}334.1^{2} \\
334.4^{3} \\
334.8^{4} \\
334.1^{8}\end{array}$ & $\begin{array}{l}364.1^{2} \\
365.5^{3} \\
367.9^{4} \\
368.4^{9}\end{array}$ & $\begin{array}{l}2.0^{2} \\
2.0^{3} \\
2.0^{4} \\
2.1^{10}\end{array}$ & $\begin{array}{c}9.2^{2} \\
10.0^{3} \\
9.8^{4} \\
11.9^{11}\end{array}$ & $\begin{array}{l}2000.4^{2} \\
2008.0^{3} \\
2012.0^{4} \\
2113.7^{12}\end{array}$ & $\begin{array}{l}37.13^{1} \\
36.94^{3} \\
37.07^{4} \\
43.23^{5}\end{array}$ \\
\hline $\begin{array}{l}\text { 2-Methylbutanoic } \\
15(4.5)\end{array}$ & $\begin{array}{l}448.0^{1} \\
447.5^{3} \\
446.8^{4} \\
448.4^{5}\end{array}$ & $\begin{array}{l}350.1^{2} \\
352.2^{3} \\
353.6^{4} \\
347.0^{5} \\
353.4^{6} \\
355.3^{7}\end{array}$ & $\begin{array}{l}347.1^{2} \\
348.9^{3} \\
349.4^{4} \\
346.0^{8}\end{array}$ & $\begin{array}{l}384.1^{2} \\
380.8^{3} \\
383.5^{4} \\
383.5^{9}\end{array}$ & $\begin{array}{l}1.6^{2} \\
1.6^{3} \\
1.6^{4} \\
1.4^{10}\end{array}$ & $\begin{array}{l}9.8^{2} \\
7.8^{3} \\
8.5^{4} \\
8.2^{I 1}\end{array}$ & $\begin{array}{l}2622.0^{2} \\
2617.0^{3} \\
2619.5^{4} \\
2729.2^{12}\end{array}$ & $\begin{array}{l}39.14^{1} \\
39.09^{3} \\
38.99^{4} \\
45.39^{5}\end{array}$ \\
\hline $\begin{array}{l}\text { 3-Methylbutanoic } \\
16(4.5)\end{array}$ & $\begin{array}{l}448.0^{1} \\
447.5^{3} \\
446.8^{4} \\
448.4^{5}\end{array}$ & $\begin{array}{l}351.1^{2} \\
352.2^{3} \\
353.6^{4} \\
343.7^{5} \\
353.4^{6} \\
355.3^{7}\end{array}$ & $\begin{array}{l}346.8^{2} \\
348.9^{3} \\
349.4^{4} \\
346.0^{8}\end{array}$ & $\begin{array}{l}385.1^{2} \\
380.8^{3} \\
383.5^{4} \\
383.5^{9}\end{array}$ & $\begin{array}{l}1.4^{2} \\
1.6^{3} \\
1.6^{4} \\
1.4^{10}\end{array}$ & $\begin{array}{l}9.8^{2} \\
7.8^{3} \\
8.5^{4} \\
8.2^{11}\end{array}$ & $\begin{array}{l}2615.3^{2} \\
2617.0^{3} \\
2619.5^{4} \\
2729.2^{12}\end{array}$ & $\begin{array}{l}39.14^{1} \\
39.09^{3} \\
38.99^{4} \\
45.39^{5}\end{array}$ \\
\hline $\begin{array}{l}\text { tret-Pentanoic } \\
\mathbf{1 7}(3.5)\end{array}$ & $\begin{array}{l}437.0^{1} \\
436.0^{3} \\
436.7^{4} \\
439.4^{5}\end{array}$ & $\begin{array}{l}337.2^{2} \\
337.7^{3} \\
337.6^{4} \\
341.4^{5} \\
345.6^{6} \\
348.0^{7}\end{array}$ & $\begin{array}{l}342.1^{2} \\
340.6^{3} \\
342.2^{4} \\
338.8^{8}\end{array}$ & $\begin{array}{l}372.1^{2} \\
373.4^{3} \\
375.8^{4} \\
375.6^{9}\end{array}$ & $\begin{array}{l}1.6^{2} \\
1.6^{3} \\
1.6^{4} \\
1.4^{10}\end{array}$ & $\begin{array}{l}8.1^{2} \\
7.8^{3} \\
8.5^{4} \\
8.2^{11}\end{array}$ & $\begin{array}{l}2569.0^{2} \\
2617.0^{3} \\
2619.5^{4} \\
2729.2^{12}\end{array}$ & $\begin{array}{l}38.46^{1} \\
37.99^{3} \\
38.04^{4} \\
44.41^{5}\end{array}$ \\
\hline
\end{tabular}


Continued Table 1

\begin{tabular}{|c|c|c|c|c|c|c|c|c|}
\hline 1 & 2 & 3 & 5 & 6 & 7 & 8 & 9 & 10 \\
\hline $\begin{array}{l}\text { 2-Methylpentanoic } \\
\mathbf{1 8}(5.5)\end{array}$ & $\begin{array}{l}467.0^{1} \\
468.0^{3} \\
466.4^{4} \\
468.6^{5}\end{array}$ & $\begin{array}{l}367.2^{3} \\
368.3^{4} \\
364.3^{5} \\
366.8^{6} \\
368.0^{7}\end{array}$ & $\begin{array}{l}364.1^{3} \\
363.0^{4} \\
357.5^{8}\end{array}$ & $\begin{array}{l}399.8^{3} \\
398.3^{4} \\
397.3^{9}\end{array}$ & $\begin{array}{l}1.3^{3} \\
1.3^{4} \\
1.2^{10}\end{array}$ & $\begin{array}{l}7.5^{3} \\
7.6^{4} \\
7.0^{11}\end{array}$ & $\begin{array}{l}3230.0^{3} \\
3227.0^{4} \\
3344.7^{12}\end{array}$ & $\begin{array}{l}40.96^{1} \\
41.06^{3} \\
40.83^{4} \\
47.58^{5}\end{array}$ \\
\hline $\begin{array}{l}\text { 2-Ethylbutanoic } \\
19(5.0)\end{array}$ & $\begin{array}{l}460.2^{2} \\
459.0^{3} \\
456.7^{4} \\
468.6^{5}\end{array}$ & $\begin{array}{c}352.2^{2} \\
359.2^{3} \\
361.1^{4} \\
360.9^{5} \\
362.0^{6} \\
363.5^{7}\end{array}$ & $\begin{array}{l}356.1^{2} \\
357.1^{3} \\
356.3^{4} \\
353.1^{8}\end{array}$ & $\begin{array}{l}395.1^{2} \\
388.1^{3} \\
391.0^{4} \\
392.3^{9}\end{array}$ & $\begin{array}{l}1.3^{2} \\
1.3^{3} \\
1.3^{4} \\
1.2^{10}\end{array}$ & $\begin{array}{l}7.7^{2} \\
7.5^{3} \\
7.6^{4} \\
7.0^{11}\end{array}$ & $\begin{array}{l}3212.0^{2} \\
3230.0^{3} \\
3227.0^{4} \\
3344.7^{12}\end{array}$ & $\begin{array}{l}40.19^{3} \\
39.92^{4} \\
47.58^{5}\end{array}$ \\
\hline $\begin{array}{l}\text { 2-Methylhexanoic } \\
\mathbf{2 0}(6.5)\end{array}$ & $\begin{array}{l}483.3^{I} \\
483.1^{2} \\
486.0^{3} \\
485.0^{4} \\
488.4^{5}\end{array}$ & $\begin{array}{l}370.7^{2} \\
381.7^{3} \\
382.0^{4} \\
378.7^{5} \\
378.2^{6} \\
378.8^{7}\end{array}$ & $\begin{array}{l}365.1^{1} \\
376.5^{3} \\
376.5^{4} \\
368.1^{8}\end{array}$ & $\begin{array}{l}401.1^{2} \\
415.8^{3} \\
412.4^{4} \\
408.9^{5}\end{array}$ & $\begin{array}{l}1.1^{2} \\
1.1^{3} \\
1.1^{4} \\
1.2^{10}\end{array}$ & $\begin{array}{l}8.0^{2} \\
7.5^{3} \\
7.6^{4} \\
7.0^{11}\end{array}$ & $\begin{array}{l}3834.0^{2} \\
3839.0^{3} \\
3834.6^{4} \\
3960.2^{12}\end{array}$ & $\begin{array}{l}42.52^{l} \\
42.79^{3} \\
42.59^{4} \\
49.76^{5}\end{array}$ \\
\hline $\begin{array}{l}\text { 3-Methylhexanoic } \\
21(6.5)\end{array}$ & $\begin{array}{l}483.3^{1} \\
486.0^{3} \\
485.0^{4} \\
488.4^{5}\end{array}$ & $\begin{array}{l}381.7^{3} \\
382.0^{4} \\
375.9^{5}\end{array}$ & $\begin{array}{l}376.5^{3} \\
375.8^{4}\end{array}$ & $\begin{array}{l}415.8^{3} \\
412.4^{4}\end{array}$ & $\begin{array}{l}1.1^{3} \\
1.1^{4} \\
1.2^{10}\end{array}$ & $\begin{array}{l}7.5^{3} \\
7.6^{4} \\
7.0^{11}\end{array}$ & $\begin{array}{c}3839.0^{3} \\
3834.6^{4} \\
3960.2^{12}\end{array}$ & $\begin{array}{l}42.52^{1} \\
42.79^{3} \\
42.59^{4} \\
49.76^{5}\end{array}$ \\
\hline $\begin{array}{l}\text { 4-Methylhexanoic } \\
22(6.5)\end{array}$ & $\begin{array}{l}490.7^{1} \\
486.0^{3} \\
485.0^{4} \\
488.4^{5}\end{array}$ & $\begin{array}{l}381.7^{3} \\
382.0^{4} \\
375.9^{5} \\
383.6^{6} \\
383.8^{7}\end{array}$ & $\begin{array}{l}376.5^{3} \\
375.8^{4} \\
373.0^{8}\end{array}$ & $\begin{array}{l}415.8^{3} \\
412.4^{4} \\
414.4^{9}\end{array}$ & $\begin{array}{l}1.1^{3} \\
1.1^{4} \\
1.2^{10}\end{array}$ & $\begin{array}{l}7.5^{3} \\
7.6^{4} \\
7.0^{11}\end{array}$ & $\begin{array}{l}3839.0^{3} \\
3834.6^{4} \\
3960.2^{12}\end{array}$ & $\begin{array}{l}43.24^{1} \\
42.79^{3} \\
42.59^{4} \\
49.76^{5}\end{array}$ \\
\hline $\begin{array}{l}\text { 5-Methylhexanoic } \\
\mathbf{2 3}(6.5)\end{array}$ & $\begin{array}{l}486.0^{1} \\
486.0^{3} \\
485.0^{4} \\
488.4^{5}\end{array}$ & $\begin{array}{l}381.7^{3} \\
382.0^{4} \\
375.9^{5} \\
380.3^{6} \\
380.7^{7}\end{array}$ & $\begin{array}{l}376.5^{3} \\
375.8^{4} \\
370.0^{8}\end{array}$ & $\begin{array}{l}415.8^{3} \\
412.4^{4} \\
411.0^{9}\end{array}$ & $\begin{array}{l}1.1^{3} \\
1.1^{4} \\
1.2^{10}\end{array}$ & $\begin{array}{l}7.5^{3} \\
7.6^{4} \\
7.0^{11}\end{array}$ & $\begin{array}{l}3839.0^{3} \\
3834.6^{4} \\
3960.2^{12}\end{array}$ & $\begin{array}{l}42.78^{1} \\
42.79^{3} \\
42.59^{4} \\
49.76^{5}\end{array}$ \\
\hline $\begin{array}{l}\text { 2.2-Dimethylpentanoic } \\
\mathbf{2 4}(5.5)\end{array}$ & $\begin{array}{l}470.0^{1} \\
468.0^{3} \\
466.4^{4} \\
481.0^{5}\end{array}$ & $\begin{array}{l}368.1^{2} \\
367.2^{3} \\
368.3^{4} \\
365.2^{5} \\
368.9^{6} \\
370.1^{7}\end{array}$ & $\begin{array}{l}365.1^{2} \\
364.1^{3} \\
363.0^{4} \\
359.5^{8}\end{array}$ & $\begin{array}{l}400.2^{2} \\
399.8^{3} \\
398.3^{4} \\
399.4^{9}\end{array}$ & $\begin{array}{l}1.1^{2} \\
1.1^{3} \\
1.1^{4} \\
1.2^{10}\end{array}$ & $\begin{array}{l}6.9^{2} \\
7.5^{3} \\
7.6^{4} \\
7.0^{11}\end{array}$ & $\begin{array}{l}3834.0^{2} \\
3839.0^{3} \\
3834.6^{4} \\
3960.2^{12}\end{array}$ & $\begin{array}{l}41.24^{1} \\
41.06^{3} \\
40.83^{4} \\
48.94^{5}\end{array}$ \\
\hline $\begin{array}{l}\text { 4.4-Dimethylpentanoic } \\
25 \text { (6.0) }\end{array}$ & $\begin{array}{l}483.3^{1} \\
477.0^{3} \\
475.8^{4} \\
481.0^{5}\end{array}$ & $\begin{array}{l}373.7^{3} \\
375.3^{4} \\
365.2^{5}\end{array}$ & $\begin{array}{l}373.7^{3} \\
369.5^{4}\end{array}$ & $\begin{array}{l}404.1^{3} \\
405.4^{4}\end{array}$ & $\begin{array}{l}1.1^{3} \\
1.1^{4} \\
1.2^{10}\end{array}$ & $\begin{array}{l}7.5^{3} \\
7.6^{4} \\
7.0^{11}\end{array}$ & $\begin{array}{c}3839.0^{3} \\
3834.6^{4} \\
3960.2^{12}\end{array}$ & $\begin{array}{l}42.52^{1} \\
41.90^{3} \\
41.72^{4} \\
48.94^{5}\end{array}$ \\
\hline $\begin{array}{l}\text { 2-Ethylpentanoic } \\
\mathbf{2 6}(6.0)\end{array}$ & $\begin{array}{l}483.3^{1} \\
477.0^{3} \\
475.8^{4} \\
482.3^{5}\end{array}$ & $\begin{array}{l}373.7^{3} \\
375.3^{4} \\
375.9^{5}\end{array}$ & $\begin{array}{l}373.7^{3} \\
369.5^{4}\end{array}$ & $\begin{array}{l}404.1^{3} \\
405.4^{4}\end{array}$ & $\begin{array}{l}1.1^{3} \\
1.1^{4} \\
1.2^{10}\end{array}$ & $\begin{array}{l}7.5^{3} \\
7.6^{4} \\
7.0^{11}\end{array}$ & $\begin{array}{c}3839.0^{3} \\
3834.6^{4} \\
3960.2^{12}\end{array}$ & $\begin{array}{l}42.52^{1} \\
41.90^{3} \\
41.72^{4} \\
48.94^{5}\end{array}$ \\
\hline $\begin{array}{l}\text { 3-Ethylpentanoic } \\
27(6.5)\end{array}$ & $\begin{array}{l}483.3^{1} \\
486.0^{3} \\
485.8^{4} \\
488.4^{5}\end{array}$ & $\begin{array}{l}381.7^{3} \\
382.0^{4} \\
375.9^{5}\end{array}$ & $\begin{array}{l}376.5^{3} \\
375.8^{4}\end{array}$ & $\begin{array}{l}415.8^{3} \\
412.4^{4}\end{array}$ & $\begin{array}{l}1.1^{3} \\
1.1^{4} \\
1.2^{10}\end{array}$ & $\begin{array}{l}7.5^{3} \\
7.6^{4} \\
7.0^{11}\end{array}$ & $\begin{array}{c}3839.0^{3} \\
3834.6^{4} \\
3960.2^{12}\end{array}$ & $\begin{array}{l}42.52^{1} \\
42.79^{3} \\
42.59^{5}\end{array}$ \\
\hline $\begin{array}{l}\text { 2-Ethyl-2-methylbutanoic } \\
\mathbf{2 8}(5.5)\end{array}$ & $\begin{array}{l}483.3^{1} \\
468.0^{3} \\
466.4^{4} \\
481.0^{5}\end{array}$ & $\begin{array}{l}367.2^{3} \\
368.3^{4} \\
365.2^{5}\end{array}$ & $\begin{array}{l}364.1^{3} \\
363.0^{4}\end{array}$ & $\begin{array}{l}399.8^{3} \\
398.3^{4}\end{array}$ & $\begin{array}{c}1.1^{3} \\
1.1^{4} \\
1.2^{10}\end{array}$ & $\begin{array}{l}7.5^{3} \\
7.6^{4} \\
7.0^{11}\end{array}$ & $\begin{array}{c}3839.0^{3} \\
3834.6^{4} \\
3960.2^{12}\end{array}$ & $\begin{array}{l}42.52^{1} \\
41.06^{3} \\
40.83^{4} \\
48.94^{5}\end{array}$ \\
\hline $\begin{array}{l}\text { 2-Methylheptanoic } \\
29(7.5)\end{array}$ & $\begin{array}{l}502.1^{1} \\
502.5^{3} \\
502.8^{4} \\
507.7^{5}\end{array}$ & $\begin{array}{l}394.2^{3} \\
394.5^{4} \\
389.7^{5}\end{array}$ & $\begin{array}{l}387.4^{3} \\
387.8^{4}\end{array}$ & $\begin{array}{l}425.9^{3} \\
425.6^{4}\end{array}$ & $\begin{array}{l}1.0^{3} \\
1.0^{4} \\
1.0^{10}\end{array}$ & $\begin{array}{l}6.4^{3} \\
6.4^{4} \\
6.7^{11}\end{array}$ & $\begin{array}{l}4448.0^{3} \\
4442.1^{4} \\
4575.7^{12}\end{array}$ & $\begin{array}{l}44.33^{1} \\
44.38^{3} \\
44.26^{4} \\
51.92^{5}\end{array}$ \\
\hline
\end{tabular}


Continued Table 1

\begin{tabular}{|c|c|c|c|c|c|c|c|c|}
\hline 1 & 2 & 3 & 5 & 6 & 7 & 8 & 9 & 10 \\
\hline $\begin{array}{l}\text { 4-Methylheptanoic } \\
30(7.5)\end{array}$ & $\begin{array}{l}502.1^{1} \\
502.5^{3} \\
502.8^{4} \\
507.7^{5}\end{array}$ & $\begin{array}{l}394.2^{3} \\
394.5^{4} \\
389.7^{5}\end{array}$ & $\begin{array}{l}387.4^{3} \\
387.8^{4}\end{array}$ & $\begin{array}{l}425.9^{3} \\
425.6^{4}\end{array}$ & $\begin{array}{l}1.0^{3} \\
1.0^{4} \\
1.0^{10}\end{array}$ & $\begin{array}{l}6.4^{3} \\
6.4^{4} \\
6.7^{11}\end{array}$ & $\begin{array}{l}4448.0^{3} \\
4442.1^{4} \\
4575.7^{12}\end{array}$ & $\begin{array}{l}44.33^{1} \\
44.38^{3} \\
44.26^{4} \\
51.92^{5}\end{array}$ \\
\hline $\begin{array}{l}\text { 6-Methylheptanoic } \\
31 \text { (7.5) }\end{array}$ & $\begin{array}{l}502.1^{1} \\
502.5^{3} \\
502.8^{4} \\
507.7^{5}\end{array}$ & $\begin{array}{l}394.2^{3} \\
394.5^{4} \\
389.7^{5}\end{array}$ & $\begin{array}{l}387.4^{3} \\
387.8^{4}\end{array}$ & $\begin{array}{l}425.9^{3} \\
425.6^{4}\end{array}$ & $\begin{array}{l}1.0^{3} \\
1.0^{4} \\
1.0^{10}\end{array}$ & $\begin{array}{l}6.4^{3} \\
6.4^{4} \\
6.7^{11}\end{array}$ & $\begin{array}{l}4448.0^{3} \\
4442.1^{4} \\
4575.7^{12}\end{array}$ & $\begin{array}{l}44.33^{1} \\
44.38^{3} \\
44.26^{4} \\
51.92^{5}\end{array}$ \\
\hline $\begin{array}{l}\text { 2.2-Dimethylhexanoic } \\
32 \text { (6.5) }\end{array}$ & $\begin{array}{l}502.1^{1} \\
477.0^{3} \\
475.8^{4} \\
500.8^{5}\end{array}$ & $\begin{array}{l}381.7^{3} \\
382.0^{4} \\
375.7^{5}\end{array}$ & $\begin{array}{l}376.5^{3} \\
375.8^{4}\end{array}$ & $\begin{array}{l}415.8^{3} \\
412.4^{4}\end{array}$ & $\begin{array}{l}1.0^{3} \\
1.0^{4} \\
1.0^{10}\end{array}$ & $\begin{array}{l}6.4^{3} \\
6.4^{4} \\
6.7^{11}\end{array}$ & $\begin{array}{l}4448.0^{3} \\
4442.1^{4} \\
4575.7^{12}\end{array}$ & $\begin{array}{l}44.33^{1} \\
42.79^{3} \\
42.59^{4} \\
51.14^{5}\end{array}$ \\
\hline $\begin{array}{l}\text { 4.4-Dimethylhexanoic } \\
33 \text { (7.0) }\end{array}$ & $\begin{array}{l}502.1^{1} \\
493.5^{3} \\
494.0^{4} \\
500.8^{5}\end{array}$ & $\begin{array}{l}388.2^{3} \\
388.4^{4} \\
375.7^{5}\end{array}$ & $\begin{array}{l}381.9^{3} \\
381.9^{4}\end{array}$ & $\begin{array}{l}420.1^{3} \\
419.1^{4}\end{array}$ & $\begin{array}{l}1.0^{3} \\
1.0^{4} \\
1.0^{10}\end{array}$ & $\begin{array}{l}6.4^{3} \\
6.4^{4} \\
6.7^{11}\end{array}$ & $\begin{array}{l}4448.0^{3} \\
4442.1^{4} \\
4575.7^{12}\end{array}$ & $\begin{array}{l}44.33^{1} \\
43.65^{3} \\
43.43^{4} \\
51.14^{5}\end{array}$ \\
\hline $\begin{array}{l}\text { 2-Ethylhexanoic } \\
\mathbf{3 4}(7.0)\end{array}$ & $\begin{array}{l}501.2^{1} \\
493.5^{3} \\
494.0^{4} \\
501.1^{5}\end{array}$ & $\begin{array}{l}383.2^{2} \\
388.2^{3} \\
388.4^{4} \\
389.7^{5} \\
391.0^{6} \\
390.9^{7}\end{array}$ & $\begin{array}{l}381.1^{2} \\
381.9^{3} \\
381.9^{4} \\
379.0^{8}\end{array}$ & $\begin{array}{l}428.1^{2} \\
420.1^{3} \\
419.1^{4} \\
422.0^{9}\end{array}$ & $\begin{array}{l}0.9^{2} \\
1.0^{3} \\
1.0^{4} \\
1.0^{10}\end{array}$ & $\begin{array}{l}8.6^{2} \\
6.4^{3} \\
6.4^{4} \\
6.7^{11}\end{array}$ & $\begin{array}{l}4448.0^{2} \\
4448.0^{3} \\
4442.1^{4} \\
4575.7^{12}\end{array}$ & $\begin{array}{l}44.25^{1} \\
43.65^{3} \\
43.43^{4} \\
51.14^{5}\end{array}$ \\
\hline $\begin{array}{l}\text { 3-Ethylhexanoic } \\
35(7.5)\end{array}$ & $\begin{array}{l}502.1^{1} \\
502.5^{3} \\
502.8^{4} \\
507.7^{5}\end{array}$ & $\begin{array}{l}394.2^{3} \\
394.5^{4} \\
389.7^{5}\end{array}$ & $\begin{array}{l}387.4^{3} \\
387.8^{4}\end{array}$ & $\begin{array}{l}425.9^{3} \\
425.6^{4}\end{array}$ & $\begin{array}{l}1.0^{3} \\
1.0^{4} \\
1.0^{10}\end{array}$ & $\begin{array}{l}6.4^{3} \\
6.4^{4} \\
6.7^{11}\end{array}$ & $\begin{array}{l}4448.0^{3} \\
4442.1^{4} \\
4575.7^{12}\end{array}$ & $\begin{array}{l}44.33^{1} \\
44.38^{3} \\
44.26^{4} \\
51.92^{5}\end{array}$ \\
\hline $\begin{array}{l}\text { 4-Ethylhexanoic } \\
36(7.5)\end{array}$ & $\begin{array}{l}502.1^{1} \\
502.5^{3} \\
502.8^{4} \\
507.7^{5}\end{array}$ & $\begin{array}{l}394.2^{3} \\
394.5^{4} \\
389.7^{5}\end{array}$ & $\begin{array}{l}387.4^{3} \\
387.8^{4}\end{array}$ & $\begin{array}{l}425.9^{3} \\
425.6^{4}\end{array}$ & $\begin{array}{l}1.0^{3} \\
1.0^{4} \\
1.0^{10}\end{array}$ & $\begin{array}{l}6.4^{3} \\
6.4^{4} \\
6.7^{11}\end{array}$ & $\begin{array}{l}4448.0^{3} \\
4442.1^{4} \\
4575.7^{12}\end{array}$ & $\begin{array}{l}44.33^{1} \\
44.38^{3} \\
44.26^{4} \\
51.92^{5}\end{array}$ \\
\hline $\begin{array}{l}\text { 2-Propylpentanoic } \\
\mathbf{3 7}(7.0)\end{array}$ & $\begin{array}{l}493.2^{1} \\
493.5^{3} \\
494.0^{4} \\
493.1^{5}\end{array}$ & $\begin{array}{l}388.2^{3} \\
388.4^{4} \\
384.2^{5} \\
385.4^{6} \\
385.6^{7}\end{array}$ & $\begin{array}{l}381.9^{3} \\
381.9^{4} \\
373.8^{8}\end{array}$ & $\begin{array}{l}420.1^{3} \\
419.1^{4} \\
416.2^{9}\end{array}$ & $\begin{array}{l}1.0^{3} \\
1.0^{4} \\
1.0^{10}\end{array}$ & $\begin{array}{l}6.4^{3} \\
6.4^{4} \\
6.7^{11}\end{array}$ & $\begin{array}{l}4448.0^{3} \\
4442.1^{4} \\
4575.7^{12}\end{array}$ & $\begin{array}{l}43.48^{1} \\
43.65^{3} \\
43.43^{4} \\
50.29^{5}\end{array}$ \\
\hline $\begin{array}{l}\text { 2-Methyloctanoic } \\
\mathbf{3 8}(8.5)\end{array}$ & $\begin{array}{l}519.6^{1} \\
518.2^{2} \\
518.6^{3} \\
519.7^{4} \\
526.5^{5}\end{array}$ & $\begin{array}{l}393.1^{2} \\
406.7^{3} \\
405.8^{4} \\
402.9^{5} \\
403.1^{6} \\
402.3^{7}\end{array}$ & $\begin{array}{l}391.1^{2} \\
398.5^{3} \\
398.8^{4} \\
389.2^{8}\end{array}$ & $\begin{array}{l}434.1^{2} \\
437.6^{3} \\
438.1^{4} \\
434.2^{9}\end{array}$ & $\begin{array}{l}0.9^{2} \\
0.9^{3} \\
0.9^{4} \\
0.9^{10}\end{array}$ & $\begin{array}{l}6.7^{2} \\
5.9^{3} \\
6.0^{4} \\
6.2^{11}\end{array}$ & $\begin{array}{l}5056.0^{2} \\
5061.0^{3} \\
5049.7^{4} \\
5191.3^{12}\end{array}$ & $\begin{array}{l}46.03^{I} \\
45.93^{3} \\
45.85^{4} \\
54.04^{5}\end{array}$ \\
\hline $\begin{array}{l}\text { 3-Methyloctanoic } \\
\text { 39 (8.5) }\end{array}$ & $\begin{array}{l}519.6^{1} \\
518.2^{2} \\
518.6^{3} \\
519.7^{4} \\
526.5^{5}\end{array}$ & $\begin{array}{l}406.7^{3} \\
405.8^{4} \\
402.9^{5}\end{array}$ & $\begin{array}{l}398.5^{3} \\
398.8^{4}\end{array}$ & $\begin{array}{l}437.6^{3} \\
438.1^{4}\end{array}$ & $\begin{array}{l}0.9^{3} \\
0.9^{4} \\
0.9^{10}\end{array}$ & $\begin{array}{l}5.9^{3} \\
6.0^{4} \\
6.2^{11}\end{array}$ & $\begin{array}{l}5061.0^{3} \\
5049.7^{4} \\
5191.3^{12}\end{array}$ & $\begin{array}{l}46.03^{1} \\
45.93^{3} \\
45.85^{4} \\
54.04^{5}\end{array}$ \\
\hline $\begin{array}{l}\text { 4-Methyloctanoic } \\
\mathbf{4 0}(8.5)\end{array}$ & $\begin{array}{l}519.6^{1} \\
518.2^{2} \\
518.6^{3} \\
519.7^{4} \\
526.5^{5}\end{array}$ & $\begin{array}{l}406.7^{3} \\
405.8^{4} \\
402.9^{5}\end{array}$ & $\begin{array}{l}398.5^{3} \\
398.8^{4}\end{array}$ & $\begin{array}{l}437.6^{3} \\
438.1^{4}\end{array}$ & $\begin{array}{l}0.9^{3} \\
0.9^{4} \\
0.9^{10}\end{array}$ & $\begin{array}{l}5.9^{3} \\
6.0^{4} \\
6.2^{11}\end{array}$ & $\begin{array}{l}5061.0^{3} \\
5049.7^{4} \\
5191.3^{12}\end{array}$ & $\begin{array}{l}46.03^{1} \\
45.93^{3} \\
45.85^{4} \\
54.04^{5}\end{array}$ \\
\hline $\begin{array}{l}\text { 6-Methyloctanoic } \\
\mathbf{4 1}(8.5)\end{array}$ & $\begin{array}{l}519.6^{1} \\
518.2^{2} \\
518.6^{3} \\
519.7^{4} \\
526.5^{5}\end{array}$ & $\begin{array}{l}406.7^{3} \\
405.8^{4} \\
402.9^{5}\end{array}$ & $\begin{array}{l}398.5^{3} \\
398.8^{4}\end{array}$ & $\begin{array}{l}437.6^{3} \\
438.1^{4}\end{array}$ & $\begin{array}{l}0.9^{3} \\
0.9^{4} \\
0.9^{10}\end{array}$ & $\begin{array}{l}5.9^{3} \\
6.0^{4} \\
6.2^{11}\end{array}$ & $\begin{array}{l}5061.0^{3} \\
5049.7^{4} \\
5191.3^{12}\end{array}$ & $\begin{array}{l}46.03^{1} \\
45.93^{3} \\
45.85^{4} \\
54.04^{5}\end{array}$ \\
\hline
\end{tabular}


Continued Table 1

\begin{tabular}{|c|c|c|c|c|c|c|c|c|}
\hline 1 & 2 & 3 & 5 & 6 & 7 & 8 & 9 & 10 \\
\hline $\begin{array}{l}\text { 7-Methyloctanoic } \\
\mathbf{4 2}(8.5)\end{array}$ & $\begin{array}{l}519.6^{1} \\
518.2^{2} \\
518.6^{3} \\
519.7^{4} \\
526.5^{5}\end{array}$ & $\begin{array}{l}406.7^{3} \\
405.8^{4} \\
402.9^{5}\end{array}$ & $\begin{array}{l}398.5^{3} \\
398.8^{4}\end{array}$ & $\begin{array}{l}437.6^{3} \\
438.1^{4}\end{array}$ & $\begin{array}{l}0.9^{3} \\
0.9^{4} \\
0.9^{10}\end{array}$ & $\begin{array}{l}5.9^{3} \\
6.0^{4} \\
6.2^{11}\end{array}$ & $\begin{array}{l}5061.0^{3} \\
5049.7^{4} \\
5191.3^{12}\end{array}$ & $\begin{array}{l}46.03^{1} \\
45.93^{3} \\
45.85^{4} \\
54.04^{5}\end{array}$ \\
\hline $\begin{array}{l}\text { 2.6-Dimethylheptanoic } \\
\mathbf{4 3}(8.0)\end{array}$ & $\begin{array}{l}519.6^{1} \\
510.0^{3} \\
511.4^{4} \\
522.8^{5}\end{array}$ & $\begin{array}{l}400.2^{3} \\
400.3^{4} \\
387.5^{5}\end{array}$ & $\begin{array}{l}392.9^{3} \\
393.4^{4}\end{array}$ & $\begin{array}{l}431.6^{3} \\
432.0^{4}\end{array}$ & $\begin{array}{l}0.9^{3} \\
0.9^{4} \\
0.9^{10}\end{array}$ & $\begin{array}{l}5.9^{3} \\
6.0^{4} \\
6.2^{11}\end{array}$ & $\begin{array}{l}5061.0^{3} \\
5049.7^{4} \\
5191.3^{12}\end{array}$ & $\begin{array}{l}46.03^{1} \\
45.10^{3} \\
45.06^{4} \\
53.61^{5}\end{array}$ \\
\hline $\begin{array}{l}\text { 2-Ethylheptanoic } \\
\mathbf{4 4}(8.0)\end{array}$ & $\begin{array}{l}519.6^{1} \\
510.0^{3} \\
511.4^{4} \\
526.5^{5}\end{array}$ & $\begin{array}{l}400.2^{3} \\
400.3^{4} \\
402.9^{5}\end{array}$ & $\begin{array}{l}392.9^{3} \\
393.4^{4}\end{array}$ & $\begin{array}{l}431.6^{3} \\
432.0^{4}\end{array}$ & $\begin{array}{l}0.9^{3} \\
0.9^{4} \\
0.9^{10}\end{array}$ & $\begin{array}{l}5.9^{3} \\
6.0^{4} \\
6.2^{11}\end{array}$ & $\begin{array}{l}5061.0^{3} \\
5049.7^{4} \\
5191.3^{12}\end{array}$ & $\begin{array}{l}46.03^{1} \\
45.10^{3} \\
45.06^{4} \\
53.61^{5}\end{array}$ \\
\hline $\begin{array}{l}\text { 3-Ethylheptanoic } \\
\mathbf{4 5}(8.5)\end{array}$ & $\begin{array}{l}519.6^{1} \\
518.6^{3} \\
519.7^{4} \\
526.5^{5}\end{array}$ & $\begin{array}{l}406.7^{3} \\
405.8^{4} \\
402.9^{5}\end{array}$ & $\begin{array}{l}398.5^{3} \\
398.8^{4}\end{array}$ & $\begin{array}{l}437.6^{3} \\
438.1^{4}\end{array}$ & $\begin{array}{l}0.9^{3} \\
0.9^{4} \\
0.9^{10}\end{array}$ & $\begin{array}{l}5.9^{3} \\
6.0^{4} \\
6.2^{11}\end{array}$ & $\begin{array}{l}5061.0^{3} \\
5049.7^{4} \\
5191.3^{12}\end{array}$ & $\begin{array}{l}46.03^{1} \\
45.93^{3} \\
45.85^{4} \\
54.04^{5}\end{array}$ \\
\hline $\begin{array}{l}\text { 2-Ethyl-3-methylhexanoic } \\
\mathbf{4 6}(7.5)\end{array}$ & $\begin{array}{l}519.6^{1} \\
502.5^{3} \\
502.8^{4} \\
504.1^{5}\end{array}$ & $\begin{array}{l}394.2^{3} \\
394.5^{4} \\
387.5^{5}\end{array}$ & $\begin{array}{l}387.4^{3} \\
387.8^{4}\end{array}$ & $\begin{array}{l}425.9^{3} \\
425.6^{4}\end{array}$ & $\begin{array}{l}0.9^{3} \\
0.9^{4} \\
0.9^{10}\end{array}$ & $\begin{array}{l}5.9^{3} \\
6.0^{4} \\
6.2^{11}\end{array}$ & $\begin{array}{l}5061.0^{3} \\
5049.7^{4} \\
5191.3^{12}\end{array}$ & $\begin{array}{l}46.03^{1} \\
44.38^{3} \\
44.26^{4} \\
51.51^{5}\end{array}$ \\
\hline $\begin{array}{l}\text { 3-Ethyl-3-methylhexanoic } \\
\mathbf{4 7}(8.0)\end{array}$ & $\begin{array}{l}519.6^{1} \\
510.0^{3} \\
511.4^{4} \\
520.1^{5}\end{array}$ & $\begin{array}{l}400.2^{3} \\
400.3^{4} \\
385.4^{5}\end{array}$ & $\begin{array}{l}392.9^{3} \\
393.4^{4}\end{array}$ & $\begin{array}{l}431.6^{3} \\
432.0^{4}\end{array}$ & $\begin{array}{l}0.9^{3} \\
0.9^{4} \\
0.9^{10}\end{array}$ & $\begin{array}{l}5.9^{3} \\
6.0^{4} \\
6.2^{11}\end{array}$ & $\begin{array}{l}5061.0^{3} \\
5049.7^{4} \\
5191.3^{12}\end{array}$ & $\begin{array}{l}46.03^{1} \\
45.10^{3} \\
45.06^{4} \\
53.31^{5}\end{array}$ \\
\hline $\begin{array}{l}\text { 3.5.5-Trimethylhexanoic } \\
\mathbf{4 8}(7.5)\end{array}$ & $\begin{array}{l}504.2^{1} \\
502.5^{3} \\
502.8^{4} \\
516.4^{5}\end{array}$ & $\begin{array}{l}394.2^{3} \\
394.5^{4} \\
382.6^{5} \\
393.2^{6} \\
393.0^{7}\end{array}$ & $\begin{array}{l}387.4^{3} \\
387.8^{4} \\
380.0^{8}\end{array}$ & $\begin{array}{l}425.9^{3} \\
425.6^{4} \\
424.1^{9}\end{array}$ & $\begin{array}{l}0.9^{3} \\
0.9^{4} \\
0.9^{10}\end{array}$ & $\begin{array}{l}5.9^{3} \\
6.0^{4} \\
6.2^{11}\end{array}$ & $\begin{array}{l}5061.0^{3} \\
5049.7^{4} \\
5191.3^{12}\end{array}$ & $\begin{array}{l}44.54^{1} \\
44.38^{3} \\
44.26^{4} \\
52.89^{5}\end{array}$ \\
\hline $\begin{array}{l}\text { 2-Propylhexanoic } \\
\mathbf{4 9}(8.0)\end{array}$ & $\begin{array}{l}519.6^{1} \\
510.0^{3} \\
511.4^{4} \\
526.5^{5}\end{array}$ & $\begin{array}{l}400.2^{3} \\
400.3^{4} \\
402.9^{5}\end{array}$ & $\begin{array}{l}392.9^{3} \\
393.4^{4}\end{array}$ & $\begin{array}{l}431.6^{3} \\
432.0^{4}\end{array}$ & $\begin{array}{l}0.9^{3} \\
0.9^{4} \\
0.9^{10}\end{array}$ & $\begin{array}{l}5.9^{3} \\
6.0^{4} \\
6.2^{11}\end{array}$ & $\begin{array}{l}5061.0^{3} \\
5049.7^{4} \\
5191.3^{12}\end{array}$ & $\begin{array}{l}46.03^{1} \\
45.10^{3} \\
45.06^{4} \\
54.04^{5}\end{array}$ \\
\hline $\begin{array}{l}\text { 2-Methylnonanoic } \\
\mathbf{5 0}(9.5)\end{array}$ & $\begin{array}{l}536.1^{1} \\
535.3^{3} \\
535.7^{4} \\
544.8^{5} \\
\end{array}$ & $\begin{array}{l}417.0^{3} \\
416.0^{4} \\
415.4^{5}\end{array}$ & $\begin{array}{l}412.4^{3} \\
409.0^{4}\end{array}$ & $\begin{array}{l}448.9^{3} \\
449.9^{4}\end{array}$ & $\begin{array}{l}0.8^{3} \\
0.8^{4} \\
0.8^{10}\end{array}$ & $\begin{array}{l}5.5^{3} \\
5.6^{4} \\
5.8^{11}\end{array}$ & $\begin{array}{c}5720.0^{3} \\
5657.2^{4} \\
5806.8^{12}\end{array}$ & $\begin{array}{l}47.63^{1} \\
47.48^{3} \\
47.35^{4} \\
56.12^{5}\end{array}$ \\
\hline $\begin{array}{l}\text { 3-Methylnonanoic } \\
\mathbf{5 1}(9.5)\end{array}$ & $\begin{array}{l}536.1^{1} \\
535.3^{3} \\
535.7^{4} \\
544.8^{5}\end{array}$ & $\begin{array}{l}417.0^{3} \\
416.0^{4} \\
415.4^{5}\end{array}$ & $\begin{array}{l}412.4^{3} \\
409.0^{4}\end{array}$ & $\begin{array}{l}448.9^{3} \\
449.9^{4}\end{array}$ & $\begin{array}{l}0.8^{3} \\
0.8^{4} \\
0.8^{10}\end{array}$ & $\begin{array}{l}5.5^{3} \\
5.6^{4} \\
5.8^{11}\end{array}$ & $\begin{array}{c}5720.0^{3} \\
5657.2^{4} \\
5806.8^{12}\end{array}$ & $\begin{array}{l}47.63^{1} \\
47.48^{3} \\
47.35^{4} \\
56.12^{5}\end{array}$ \\
\hline $\begin{array}{l}\text { 4-Methylnonanoic } \\
\mathbf{5 2}(9.5)\end{array}$ & $\begin{array}{l}536.1^{1} \\
535.3^{3} \\
535.7^{4} \\
544.8^{5}\end{array}$ & $\begin{array}{l}417.0^{3} \\
416.0^{4} \\
415.4^{5}\end{array}$ & $\begin{array}{l}412.4^{3} \\
409.0^{4}\end{array}$ & $\begin{array}{l}448.9^{3} \\
449.9^{4}\end{array}$ & $\begin{array}{l}0.8^{3} \\
0.8^{4} \\
0.8^{10}\end{array}$ & $\begin{array}{l}5.5^{3} \\
5.6^{4} \\
5.8^{11}\end{array}$ & $\begin{array}{c}5720.0^{3} \\
5657.2^{4} \\
5806.8^{12}\end{array}$ & $\begin{array}{l}47.63^{1} \\
47.48^{3} \\
47.35^{4} \\
56.12^{5}\end{array}$ \\
\hline $\begin{array}{l}\text { 8-Methylnonanoic } \\
\mathbf{5 3}(9.5)\end{array}$ & $\begin{array}{l}536.1^{1} \\
535.3^{3} \\
535.7^{4} \\
544.8^{5}\end{array}$ & $\begin{array}{l}417.0^{3} \\
416.0^{4} \\
415.4^{5}\end{array}$ & $\begin{array}{l}412.4^{3} \\
409.0^{4}\end{array}$ & $\begin{array}{l}448.9^{3} \\
449.9^{4}\end{array}$ & $\begin{array}{l}0.8^{3} \\
0.8^{4} \\
0.8^{10}\end{array}$ & $\begin{array}{l}5.5^{3} \\
5.6^{4} \\
5.8^{11}\end{array}$ & $\begin{array}{c}5720.0^{3} \\
5657.2^{4} \\
5806.8^{12}\end{array}$ & $\begin{array}{l}47.63^{1} \\
47.48^{3} \\
47.35^{4} \\
56.12^{5}\end{array}$ \\
\hline $\begin{array}{l}\text { 2.2-Dimethyloctanoic } \\
\mathbf{5 4}(8.5)\end{array}$ & $\begin{array}{l}536.1^{1} \\
518.6^{3} \\
519.7^{4} \\
538.7^{5}\end{array}$ & $\begin{array}{l}406.7^{3} \\
405.8^{4} \\
394.4^{6}\end{array}$ & $\begin{array}{l}398.5^{3} \\
398.8^{4}\end{array}$ & $\begin{array}{l}437.6^{3} \\
438.1^{4}\end{array}$ & $\begin{array}{l}0.8^{3} \\
0.8^{4} \\
0.8^{10}\end{array}$ & $\begin{array}{l}5.5^{3} \\
5.6^{4} \\
5.8^{11}\end{array}$ & $\begin{array}{c}5720.0^{3} \\
5657.2^{4} \\
5806.8^{12}\end{array}$ & $\begin{array}{l}47.63^{1} \\
45.93^{3} \\
45.85^{4} \\
55.42^{5}\end{array}$ \\
\hline
\end{tabular}


Continued Table 1

\begin{tabular}{|c|c|c|c|c|c|c|c|c|}
\hline 1 & 2 & 3 & 5 & 6 & 7 & 8 & 9 & 10 \\
\hline $\begin{array}{l}\text { 2-Ethyloctanoic } \\
\mathbf{5 5}(9.0)\end{array}$ & $\begin{array}{l}536.1^{1} \\
527.2^{3} \\
527.8^{4} \\
544.8^{5}\end{array}$ & $\begin{array}{l}413.2^{3} \\
411.1^{4} \\
415.4^{5}\end{array}$ & $\begin{array}{l}404.1^{3} \\
404.0^{4}\end{array}$ & $\begin{array}{l}443.6^{3} \\
444.1^{4}\end{array}$ & $\begin{array}{l}0.8^{3} \\
0.8^{4} \\
0.8^{10}\end{array}$ & $\begin{array}{l}5.5^{3} \\
5.6^{4} \\
5.8^{11}\end{array}$ & $\begin{array}{l}5720.0^{3} \\
5657.2^{4} \\
5806.8^{12}\end{array}$ & $\begin{array}{l}47.63^{1} \\
46.76^{3} \\
46.61^{4} \\
56.12^{5}\end{array}$ \\
\hline $\begin{array}{l}\text { 4-Ethyloctanoic } \\
\mathbf{5 6}(9.5)\end{array}$ & $\begin{array}{l}536.1^{1} \\
535.3^{3} \\
535.7^{4} \\
544.8^{5}\end{array}$ & $\begin{array}{l}417.0^{3} \\
416.0^{4} \\
415.4^{5}\end{array}$ & $\begin{array}{l}412.4^{3} \\
409.0^{4}\end{array}$ & $\begin{array}{l}448.9^{3} \\
449.9^{4}\end{array}$ & $\begin{array}{l}0.8^{3} \\
0.8^{4} \\
0.8^{10}\end{array}$ & $\begin{array}{l}5.5^{3} \\
5.6^{4} \\
5.8^{11}\end{array}$ & $\begin{array}{l}5720.0^{3} \\
5657.2^{4} \\
5806.8^{12}\end{array}$ & $\begin{array}{l}47.63^{1} \\
47.48^{3} \\
47.35^{4} \\
56.12^{5}\end{array}$ \\
\hline $\begin{array}{l}\text { 2-Propylheptanoic } \\
\mathbf{5 7}(9.0)\end{array}$ & $\begin{array}{l}536.1^{1} \\
527.2^{3} \\
527.8^{4} \\
544.8^{5}\end{array}$ & $\begin{array}{l}413.2^{3} \\
411.1^{4} \\
415.4^{5}\end{array}$ & $\begin{array}{l}404.1^{3} \\
404.0^{4}\end{array}$ & $\begin{array}{l}443.6^{3} \\
444.1^{4}\end{array}$ & $\begin{array}{l}0.8^{3} \\
0.8^{4} \\
0.8^{10}\end{array}$ & $\begin{array}{l}5.5^{3} \\
5.6^{4} \\
5.8^{11}\end{array}$ & $\begin{array}{l}5720.0^{3} \\
5657.2^{4} \\
5806.8^{12}\end{array}$ & $\begin{array}{l}47.63^{1} \\
46.76^{3} \\
46.61^{4} \\
56.12^{5}\end{array}$ \\
\hline $\begin{array}{l}\text { 2.5-Dimethyl-2-ethyl- } \\
\text { hexanoic } \\
\mathbf{5 8}(8.0)\end{array}$ & $\begin{array}{l}536.1^{1} \\
510.0^{3} \\
511.4^{4} \\
535.5^{5}\end{array}$ & $\begin{array}{l}400.2^{3} \\
400.3^{4} \\
400.3^{5}\end{array}$ & $\begin{array}{l}392.9^{3} \\
393.4^{4}\end{array}$ & $\begin{array}{l}431.6^{3} \\
432.0^{4}\end{array}$ & $\begin{array}{l}0.8^{3} \\
0.8^{4} \\
0.8^{10}\end{array}$ & $\begin{array}{l}5.5^{3} \\
5.6^{4} \\
5.8^{11}\end{array}$ & $\begin{array}{l}5720.0^{3} \\
5657.2^{4} \\
5806.8^{12}\end{array}$ & $\begin{array}{l}47.63^{1} \\
45.10^{3} \\
45.06^{4} \\
57.12^{5}\end{array}$ \\
\hline $\begin{array}{l}\text { tret-Decanoic } \\
\mathbf{5 9}(9.0)\end{array}$ & $\begin{array}{l}536.1^{1} \\
527.2^{3} \\
527.8^{4} \\
538.7^{5}\end{array}$ & $\begin{array}{l}413.2^{3} \\
411.1^{4} \\
394.4^{5}\end{array}$ & $\begin{array}{l}404.1^{3} \\
404.0^{4}\end{array}$ & $\begin{array}{l}443.6^{3} \\
444.1^{4}\end{array}$ & $\begin{array}{l}0.8^{3} \\
0.8^{4} \\
0.8^{10}\end{array}$ & $\begin{array}{l}5.5^{3} \\
5.6^{4} \\
5.8^{11}\end{array}$ & $\begin{array}{l}5720.0^{3} \\
5657.2^{4} \\
5806.8^{12}\end{array}$ & $\begin{array}{l}47.63^{1} \\
46.76^{3} \\
46.61^{4} \\
55.42^{5}\end{array}$ \\
\hline $\begin{array}{l}\text { 2-Methyldecanoic } \\
\mathbf{6 0}(10.5)\end{array}$ & $\begin{array}{l}551.8^{1} \\
550.3^{3} \\
550.7^{4} \\
562.5^{5}\end{array}$ & $\begin{array}{l}424.4^{3} \\
425.1^{4} \\
427.4^{5}\end{array}$ & $\begin{array}{l}421.2^{3} \\
418.3^{4}\end{array}$ & $\begin{array}{l}459.4^{3} \\
460.8^{4}\end{array}$ & $\begin{array}{l}0.7^{3} \\
0.7^{4} \\
0.7^{10}\end{array}$ & $\begin{array}{l}5.2^{3} \\
5.3^{3} \\
5.4^{11}\end{array}$ & $\begin{array}{c}6253.0^{3} \\
6264.7^{4} \\
6422.3^{12}\end{array}$ & $\begin{array}{l}49.15^{1} \\
48.94^{3} \\
48.77^{4} \\
58.16^{5}\end{array}$ \\
\hline $\begin{array}{l}\text { 3-Methyldecanoic } \\
\mathbf{6 1}(10.5)\end{array}$ & $\begin{array}{l}551.8^{1} \\
550.3^{3} \\
550.7^{4} \\
562.5^{5}\end{array}$ & $\begin{array}{l}424.4^{3} \\
425.1^{4} \\
427.4^{5}\end{array}$ & $\begin{array}{l}421.2^{3} \\
418.3^{4}\end{array}$ & $\begin{array}{l}459.4^{3} \\
460.8^{4}\end{array}$ & $\begin{array}{l}0.7^{3} \\
0.7^{4} \\
0.7^{10}\end{array}$ & $\begin{array}{l}5.2^{3} \\
5.3^{3} \\
5.4^{11}\end{array}$ & $\begin{array}{c}6253.0^{3} \\
6264.7^{4} \\
6422.3^{12}\end{array}$ & $\begin{array}{l}49.15^{1} \\
48.94^{3} \\
48.77^{4} \\
58.16^{5}\end{array}$ \\
\hline $\begin{array}{l}\text { 6-Methyldecanoic } \\
\mathbf{6 2}(10.5)\end{array}$ & $\begin{array}{l}551.8^{1} \\
550.3^{3} \\
550.7^{4} \\
562.5^{5}\end{array}$ & $\begin{array}{l}424.4^{3} \\
425.1^{4} \\
427.4^{5}\end{array}$ & $\begin{array}{l}421.2^{3} \\
418.3^{4}\end{array}$ & $\begin{array}{l}459.4^{3} \\
460.8^{4}\end{array}$ & $\begin{array}{l}0.7^{3} \\
0.7^{4} \\
0.7^{10}\end{array}$ & $\begin{array}{l}5.2^{3} \\
5.3^{3} \\
5.4^{11}\end{array}$ & $\begin{array}{c}6253.0^{3} \\
6264.7^{4} \\
6422.3^{12}\end{array}$ & $\begin{array}{l}49.15^{1} \\
48.94^{3} \\
48.77^{4} \\
58.16^{5}\end{array}$ \\
\hline $\begin{array}{l}\text { 8-Methyldecanoic } \\
\mathbf{6 3}(10.5)\end{array}$ & $\begin{array}{l}551.8^{1} \\
550.3^{3} \\
550.7^{4} \\
562.5^{5}\end{array}$ & $\begin{array}{l}424.4^{3} \\
425.1^{4} \\
427.4^{5}\end{array}$ & $\begin{array}{l}421.2^{3} \\
418.3^{4}\end{array}$ & $\begin{array}{l}459.4^{3} \\
460.8^{4}\end{array}$ & $\begin{array}{l}0.7^{3} \\
0.7^{4} \\
0.7^{10}\end{array}$ & $\begin{array}{l}5.2^{3} \\
5.3^{3} \\
5.4^{11}\end{array}$ & $\begin{array}{c}6253.0^{3} \\
6264.7^{4} \\
6422.3^{12}\end{array}$ & $\begin{array}{l}49.15^{1} \\
48.94^{3} \\
48.77^{4} \\
58.16^{5}\end{array}$ \\
\hline $\begin{array}{l}\text { 2.2-Dimethylnonanoic } \\
\mathbf{6 4}(9.5)\end{array}$ & $\begin{array}{l}551.8^{1} \\
535.3^{3} \\
535.7^{4} \\
556.8^{5}\end{array}$ & $\begin{array}{l}417.0^{3} \\
416.0^{4} \\
402.8^{5}\end{array}$ & $\begin{array}{l}412.4^{3} \\
409.0^{4}\end{array}$ & $\begin{array}{l}448.9^{3} \\
449.9^{4}\end{array}$ & $\begin{array}{l}0.7^{3} \\
0.7^{4} \\
0.7^{10}\end{array}$ & $\begin{array}{l}5.2^{3} \\
5.3^{3} \\
5.4^{11}\end{array}$ & $\begin{array}{c}6253.0^{3} \\
6264.7^{4} \\
6422.3^{12}\end{array}$ & $\begin{array}{l}49.15^{1} \\
47.48^{3} \\
47.35^{4} \\
57.50^{5}\end{array}$ \\
\hline $\begin{array}{l}\text { 2-Ethylnonanoic } \\
\mathbf{6 5}(10.0)\end{array}$ & $\begin{array}{l}551.8^{1} \\
543.4^{3} \\
543.3^{4} \\
562.5^{5}\end{array}$ & $\begin{array}{l}420.7^{3} \\
420.7^{4} \\
427.4^{5}\end{array}$ & $\begin{array}{l}420.7^{3} \\
413.8^{4}\end{array}$ & $\begin{array}{l}454.1^{3} \\
455.4^{4}\end{array}$ & $\begin{array}{l}0.7^{3} \\
0.7^{4} \\
0.7^{10}\end{array}$ & $\begin{array}{l}5.2^{3} \\
5.3^{3} \\
5.4^{11}\end{array}$ & $\begin{array}{l}6253.0^{3} \\
6264.7^{4} \\
6422.3^{12}\end{array}$ & $\begin{array}{l}49.15^{1} \\
48.20^{3} \\
48.07^{4} \\
58.16^{5}\end{array}$ \\
\hline $\begin{array}{l}\text { 2-Methylundecanoic } \\
\mathbf{6 6}(11.5)\end{array}$ & $\begin{array}{l}566.5^{1} \\
564.1^{3} \\
564.9^{4} \\
579.7^{5}\end{array}$ & $\begin{array}{l}432.6^{3} \\
433.0^{4} \\
438.9^{5}\end{array}$ & $\begin{array}{l}425.7^{3} \\
426.7^{4}\end{array}$ & $\begin{array}{l}470.6^{3} \\
471.0^{4}\end{array}$ & $\begin{array}{l}0.6^{3} \\
0.6^{4} \\
0.7^{10}\end{array}$ & $\begin{array}{l}5.1^{3} \\
5.0^{4} \\
5.1^{11}\end{array}$ & $\begin{array}{l}6850.0^{3} \\
6872.3^{4} \\
7037.8^{12}\end{array}$ & $\begin{array}{l}50.59^{1} \\
50.36^{3} \\
50.10^{4} \\
60.17^{5}\end{array}$ \\
\hline $\begin{array}{l}\text { 3-Methylundecanoic } \\
67 \text { (11.5) }\end{array}$ & $\begin{array}{l}566.5^{1} \\
564.1^{3} \\
564.9^{4} \\
579.7^{5}\end{array}$ & $\begin{array}{l}432.6^{3} \\
433.0^{4} \\
438.9^{5}\end{array}$ & $\begin{array}{l}425.7^{3} \\
426.7^{4}\end{array}$ & $\begin{array}{l}470.6^{3} \\
471.0^{4}\end{array}$ & $\begin{array}{l}0.6^{3} \\
0.6^{4} \\
0.7^{10}\end{array}$ & $\begin{array}{l}5.1^{3} \\
5.0^{4} \\
5.1^{11}\end{array}$ & $\begin{array}{l}6850.0^{3} \\
6872.3^{4} \\
7037.8^{12}\end{array}$ & $\begin{array}{l}50.59^{1} \\
50.36^{3} \\
50.10^{4} \\
60.17^{5}\end{array}$ \\
\hline $\begin{array}{l}\text { 10-Methylundecanoic } \\
68 \text { (11.5) }\end{array}$ & $\begin{array}{l}566.5^{1} \\
564.1^{3} \\
564.9^{4} \\
579.7^{5}\end{array}$ & $\begin{array}{l}432.6^{3} \\
433.0^{4} \\
438.9^{5}\end{array}$ & $\begin{array}{l}425.7^{3} \\
426.7^{4}\end{array}$ & $\begin{array}{l}470.6^{3} \\
471.0^{4}\end{array}$ & $\begin{array}{l}0.6^{3} \\
0.6^{4} \\
0.7^{10}\end{array}$ & $\begin{array}{l}5.1^{3} \\
5.0^{4} \\
5.1^{11}\end{array}$ & $\begin{array}{c}6850.0^{3} \\
6872.3^{4} \\
7037.8^{12}\end{array}$ & $\begin{array}{l}50.59^{1} \\
50.36^{3} \\
50.10^{4} \\
60.17^{5}\end{array}$ \\
\hline
\end{tabular}


Continued Table 1

\begin{tabular}{|c|c|c|c|c|c|c|c|c|}
\hline 1 & 2 & 3 & 5 & 6 & 7 & 8 & 9 & 10 \\
\hline $\begin{array}{l}\text { 2.2-Dimethyldecanoic } \\
69(10.5)\end{array}$ & $\begin{array}{l}566.5^{1} \\
550.3^{3} \\
550.7^{4} \\
579.7^{5}\end{array}$ & $\begin{array}{l}424.4^{3} \\
425.1^{4} \\
410.4^{5}\end{array}$ & $\begin{array}{l}420.7^{3} \\
413.8^{4}\end{array}$ & $\begin{array}{l}459.4^{3} \\
460.8^{4}\end{array}$ & $\begin{array}{l}0.6^{3} \\
0.6^{4} \\
0.7^{10}\end{array}$ & $\begin{array}{l}5.1^{3} \\
5.0^{4} \\
5.1^{11}\end{array}$ & $\begin{array}{l}6850.0^{3} \\
6872.3^{4} \\
7037.8^{12}\end{array}$ & $\begin{array}{l}50.59^{1} \\
48.94^{3} \\
48.77^{4} \\
59.53^{5}\end{array}$ \\
\hline $\begin{array}{l}\text { 2-Ethyldecanoic } \\
70(11.0)\end{array}$ & $\begin{array}{c}566.5^{1} \\
564.1^{3} \\
564.9^{4} \\
579.7^{5}\end{array}$ & $\begin{array}{l}429.2^{3} \\
429.2^{4} \\
438.9^{5}\end{array}$ & $\begin{array}{l}421.2^{3} \\
422.6^{4}\end{array}$ & $\begin{array}{l}459.4^{3} \\
466.0^{4}\end{array}$ & $\begin{array}{l}0.6^{3} \\
0.6^{4} \\
0.7^{10}\end{array}$ & $\begin{array}{l}5.1^{3} \\
5.0^{4} \\
5.1^{11}\end{array}$ & $\begin{array}{l}6850.0^{3} \\
6872.3^{4} \\
7037.8^{12}\end{array}$ & $\begin{array}{l}50.59^{1} \\
49.00^{3} \\
49.44^{4} \\
60.17^{5}\end{array}$ \\
\hline $\begin{array}{l}\text { 2-Butyloctanoic } \\
71(11.0)\end{array}$ & $\begin{array}{c}566.5^{1} \\
564.1^{3} \\
564.9^{4} \\
579.7^{5}\end{array}$ & $\begin{array}{l}429.2^{3} \\
429.2^{4} \\
438.9^{5}\end{array}$ & $\begin{array}{l}421.2^{3} \\
422.6^{4}\end{array}$ & $\begin{array}{l}459.4^{3} \\
466.0^{4}\end{array}$ & $\begin{array}{l}0.6^{3} \\
0.6^{4} \\
0.7^{10}\end{array}$ & $\begin{array}{l}5.1^{3} \\
5.0^{4} \\
5.1^{11}\end{array}$ & $\begin{array}{l}6850.0^{3} \\
6872.3^{4} \\
7037.8^{12}\end{array}$ & $\begin{array}{l}50.59^{1} \\
49.00^{3} \\
49.44^{4} \\
60.17^{5}\end{array}$ \\
\hline $\begin{array}{l}\text { 2-Methyldodecanoic } \\
72 \text { (12.5) }\end{array}$ & $\begin{array}{l}580.6^{1} \\
578.2^{3} \\
578.2^{4} \\
596.5^{5}\end{array}$ & $\begin{array}{l}439.6^{3} \\
439.8^{4} \\
449.8^{5}\end{array}$ & $\begin{array}{l}434.4^{3} \\
434.3^{4}\end{array}$ & $\begin{array}{l}480.9^{3} \\
480.4^{4}\end{array}$ & $\begin{array}{l}0.6^{3} \\
0.6^{4} \\
0.6^{10}\end{array}$ & $\begin{array}{l}4.9^{3} \\
4.8^{4} \\
4.8^{11}\end{array}$ & $\begin{array}{c}7453.0^{3} \\
7479.8^{4} \\
7653.4^{12}\end{array}$ & $\begin{array}{l}51.97^{1} \\
49.00^{3} \\
51.35^{4} \\
62.14^{5}\end{array}$ \\
\hline $\begin{array}{l}\text { 3-Methyldodecanoic } \\
73(12.5)\end{array}$ & $\begin{array}{l}580.6^{1} \\
578.2^{3} \\
578.2^{4} \\
596.5^{5}\end{array}$ & $\begin{array}{l}439.6^{3} \\
439.8^{4} \\
449.8^{5}\end{array}$ & $\begin{array}{l}434.4^{3} \\
434.3^{4}\end{array}$ & $\begin{array}{l}480.9^{3} \\
480.4^{4}\end{array}$ & $\begin{array}{l}0.6^{3} \\
0.6^{4} \\
0.6^{10}\end{array}$ & $\begin{array}{l}4.9^{3} \\
4.8^{4} \\
4.8^{11}\end{array}$ & $\begin{array}{c}7453.0^{3} \\
7479.8^{4} \\
7653.4^{12}\end{array}$ & $\begin{array}{l}51.97^{1} \\
49.00^{3} \\
51.35^{4} \\
62.14^{5}\end{array}$ \\
\hline $\begin{array}{l}\text { 10-Methyldodecanoic } \\
74(12.5)\end{array}$ & $\begin{array}{l}580.6^{1} \\
578.2^{3} \\
578.2^{4} \\
596.5^{5}\end{array}$ & $\begin{array}{l}439.6^{3} \\
439.8^{4} \\
449.8^{5}\end{array}$ & $\begin{array}{l}434.4^{3} \\
434.3^{4}\end{array}$ & $\begin{array}{l}480.9^{3} \\
480.4^{4}\end{array}$ & $\begin{array}{l}0.6^{3} \\
0.6^{4} \\
0.6^{10}\end{array}$ & $\begin{array}{l}4.9^{3} \\
4.8^{4} \\
4.8^{11}\end{array}$ & $\begin{array}{c}7453.0^{3} \\
7479.8^{4} \\
7653.4^{12}\end{array}$ & $\begin{array}{l}51.97^{1} \\
49.00^{3} \\
51.35^{4} \\
62.14^{5}\end{array}$ \\
\hline $\begin{array}{l}\text { 11-Methyldodecanoic } \\
75 \text { (12.5) }\end{array}$ & $\begin{array}{l}580.6^{1} \\
578.2^{3} \\
578.2^{4} \\
596.5^{5}\end{array}$ & $\begin{array}{l}439.6^{3} \\
439.8^{4} \\
449.8^{5}\end{array}$ & $\begin{array}{l}434.4^{3} \\
434.3^{4}\end{array}$ & $\begin{array}{l}480.9^{3} \\
480.4^{4}\end{array}$ & $\begin{array}{l}0.6^{3} \\
0.6^{4} \\
0.6^{10}\end{array}$ & $\begin{array}{l}4.9^{3} \\
4.8^{4} \\
4.8^{11}\end{array}$ & $\begin{array}{c}7453.0^{3} \\
7479.8^{4} \\
7653.4^{12}\end{array}$ & $\begin{array}{l}51.97^{1} \\
49.00^{3} \\
51.35^{4} \\
62.14^{5}\end{array}$ \\
\hline $\begin{array}{l}\text { 2.2-Dimethylundecanoic } \\
76 \text { (11.5) }\end{array}$ & $\begin{array}{c}580.6^{1} \\
564.1^{3} \\
564.9^{4} \\
591.2^{5}\end{array}$ & $\begin{array}{l}432.6^{3} \\
433.0^{4} \\
417.5^{5}\end{array}$ & $\begin{array}{l}425.7^{3} \\
426.7^{4}\end{array}$ & $\begin{array}{l}470.6^{3} \\
471.0^{4}\end{array}$ & $\begin{array}{l}0.6^{3} \\
0.6^{4} \\
0.6^{10}\end{array}$ & $\begin{array}{l}4.9^{3} \\
4.8^{4} \\
4.8^{11}\end{array}$ & $\begin{array}{c}7453.0^{3} \\
7479.8^{4} \\
7653.4^{12}\end{array}$ & $\begin{array}{l}51.97^{1} \\
50.36^{3} \\
50.10^{4} \\
61.53^{5}\end{array}$ \\
\hline $\begin{array}{l}\text { 2-Ethylundecanoic } \\
77(12.0)\end{array}$ & $\begin{array}{l}580.6^{1} \\
571.0^{3} \\
571.7^{4} \\
596.5^{5}\end{array}$ & $\begin{array}{l}437.1^{3} \\
436.5^{4} \\
449.8^{5}\end{array}$ & $\begin{array}{l}429.7^{3} \\
430.6^{4}\end{array}$ & $\begin{array}{l}476.6^{3} \\
475.8^{4}\end{array}$ & $\begin{array}{l}0.6^{3} \\
0.6^{4} \\
0.6^{10}\end{array}$ & $\begin{array}{l}4.9^{3} \\
4.8^{4} \\
4.8^{11}\end{array}$ & $\begin{array}{l}7453.0^{3} \\
7479.8^{4} \\
7653.4^{12}\end{array}$ & $\begin{array}{l}51.97^{1} \\
51.03^{3} \\
50.73^{4} \\
62.14^{5}\end{array}$ \\
\hline
\end{tabular}

Notes. ${ }^{1,2}$ Literature data[3,4]. ${ }^{3-5}$ The calculated values by CCR1, the CCR2 and ACD/Lab. ${ }^{6-8}$ Prediction by equations 9-13.

We are developing a new method for the prediction of physico-chemical and fire hazard indices, which is called "the carbon chain rules" (hereinafter referred to as CCR). The approaches of descriptor and comparative methods are combined in the CCR. There are two variants of the CCR: the manual option or the CCR1 and the not manual option or the CCR2. The first one includes the evaluation of properties of substances as the arithmetic average between the values of the indices of the nearest neighbors of homological series, and the CCR2, which involves the use of equations based on the descriptor of the CCC (conventional carbon chain). The CCR framework and the order of application of the CCR1 and the CCR2 methods with examples are given in previously issued material (see [1, $5]$ and references in these articles). The studied group of compounds was divided into training and control groups to derive equations for CCR2. In the first sample the normal compounds (1)-(13) were included, and the second one consisted of compounds with isomeric structures. As a result of mathematical processing of the training sample data using the M. Excel 2010 program, equations 
(1)-(8) for predicting the boiling and the flash points, the temperature and concentration flammability limits, the heat of combustion and vaporization of carboxylic acids were obtained (Table 2). According to the equations mentioned above the forecast of physico-chemical and fire hazard characteristics of carboxylic acids was performed (Table 1).

The following feature of CCR to predict the properties of organic compounds effectively, which can be described by the general formula $\mathrm{R}-\mathrm{F}$, where $\mathrm{R}$ is an alkyl substituent, and $\mathrm{F}$ is a functional or pseudo-functional group, is revealed as a result of work with CCR. A fragment of a molecule can

Table 2. The methods of CCR1, CCR2 and the comparison equations

\begin{tabular}{|c|c|c|c|c|c|c|c|}
\hline Formula / Method & No. & $\mathrm{n}$ & $r$ & RMSE & AARD & $\varepsilon$ & $\sigma$ \\
\hline CCR1 $(N B P)$ & - & 27 & 0.9989 & 2.30 & 0.35 & 1.68 & 1.59 \\
\hline$T B=-0.4485(\mathrm{CCC})^{2}+24.046(\mathrm{CCC})+347.7(\mathrm{CCR} 2)$ & (1) & 27 & 0.9985 & 2.65 & 0.41 & 1.93 & 1.84 \\
\hline $\mathrm{ACD} / \mathrm{Lab}(N B P)$ & - & 27 & 0.9963 & 4.26 & 0.54 & 2.64 & 3.41 \\
\hline CCR1 $(F P)$ & - & 22 & 0.9980 & 2.43 & 0.54 & 1.98 & 1.37 \\
\hline $\begin{array}{l}F P=-0.572(\mathrm{CCC})^{2}+20.5(\mathrm{CCC})+272.9 \\
(\mathrm{CCR} 2)\end{array}$ & (2) & 22 & 0.9985 & 2.35 & 0.36 & 1.66 & 1.65 \\
\hline $\mathrm{ACD} / \mathrm{Lab}(F P)$ & - & 22 & 0.9481 & 15.97 & 2.61 & 9.37 & 2.49 \\
\hline$F P=a \cdot N B P+b$ & (9) & 22 & 0.9954 & 3.74 & 0.74 & 2.78 & 2.49 \\
\hline$F P=a_{0}+a_{i} \cdot N B P+\sum a_{i} \cdot l_{i}$ & (10a) & 22 & 0.9952 & 4.36 & 0.94 & 3.38 & 2.73 \\
\hline CCR1 $(L F T L)$ & - & 19 & 0.9984 & 4.56 & 0.97 & 3.73 & 2.70 \\
\hline$L F T L=-0.4374(\mathrm{CCC})^{2}+18.049(\mathrm{CCC})+277.0(\mathrm{CCR} 2)$ & (3) & 19 & 0.9995 & 1.22 & 0.27 & 0.99 & 0.74 \\
\hline$L F T L=a_{0}+a_{i} \cdot N B P+\sum a_{i} \cdot l_{i}$ & $(10 b)$ & 18 & 0.9991 & 5.48 & 1.13 & 4.41 & 3.35 \\
\hline CCR1 (UFTL) & - & 20 & 0.9962 & 3.67 & 0.70 & 2.82 & 2.42 \\
\hline$U F T L=-0.3875(\mathrm{CCC})^{2}+18.698(\mathrm{CCC})+307.2(\mathrm{CCR} 2)$ & (4) & 20 & 0.9966 & 3.28 & 0.63 & 2.50 & 2.17 \\
\hline$U F T L=a_{0}+a_{i} \cdot t_{\text {кии }}+\sum a_{i} \cdot l_{i}$ & $(10 \mathrm{c})$ & 19 & 0.9977 & 3.30 & 0.69 & 2.83 & 1.75 \\
\hline $\operatorname{CCR} 1(L F L)$ & - & 21 & 0.9936 & 0.09 & 3.95 & 0.05 & 0.08 \\
\hline$L F L=-0.47+\frac{16.552}{N_{c}}-\frac{39.362}{N_{c}^{2}}+\mathrm{e}^{-N_{c}}$ & (5) & 21 & 0.9984 & 0.05 & 2.73 & 0.03 & 0.04 \\
\hline$L F L=100 / \sum_{\mathrm{s}=1}^{\mathrm{q}} h_{s} m_{s}$ & (11) & 21 & 0.9888 & 0.16 & 6.49 & 0.10 & 0.13 \\
\hline CCR1 $(U F L)$ & - & 15 & 0.9861 & 0.34 & 3.32 & 0.25 & 0.24 \\
\hline$U F L=22.671 \times N_{c}^{-0.608}$ & (6) & 15 & 0.9835 & 0.35 & 3.40 & 0.26 & 0.25 \\
\hline $\begin{array}{c}C_{6}=100\left(\sum_{j=1}^{l} h_{j} m_{j}+\sum_{s=1}^{q} q_{s}\right) \quad n p u \beta \leq 8 \\
C_{\beta}=100 /(0,768 \beta+6,554) \quad \text { при } \beta>8\end{array}$ & $\begin{array}{l}(12 a) \\
(12 b)\end{array}$ & 15 & 0.9689 & 1.38 & 9.67 & 0.86 & 1.12 \\
\hline CCR1 $\left(H_{\text {comb }}\right)$ & - & & 0.9999 & 20.00 & 0.32 & 11.30 & 16.91 \\
\hline$H_{c o m b}=-607.54 \times N_{c}+418.2$ & (7) & & 0.9999 & 20.37 & 0.40 & 12.93 & 16.13 \\
\hline$H_{\text {comb }}=-(339.4 C+1257 H-108.9 O-225.9 H)(\mathrm{kJ} / \mathrm{kg})$ & $(13)$ & & 0.9999 & 131.55 & 4.17 & 128.47 & 28.99 \\
\hline CCR1 $\left(H_{\text {vap }}\right)$ & - & 21 & 0.9968 & 0.38 & 0.56 & 0.25 & 0.30 \\
\hline$H_{\text {vap }}=-0.0422(\mathrm{CCC})^{2}+2.2618(\mathrm{CCC})+29.67(\mathrm{CCR} 2)$ & $(8)$ & 21 & 0.9973 & 0.37 & 0.64 & 0.28 & 0.26 \\
\hline $\mathrm{ACD} / \mathrm{Lab}\left(H_{v a p}\right)$ & - & 21 & 0.9008 & 6.31 & 14.79 & 6.15 & 1.43 \\
\hline
\end{tabular}


be considered as a pseudo-functional group [6,7]. Usually the physicochemical properties of the first member of homologous series differ substantially from the properties of the subsequent compounds $[8,9]$. Carboxylic acids are no exception. In particular, pKa formic acid (1) is about 1.4 times less than that of other monoacids because of the strong effect of the carboxyl group [10]. Therefore, it is not surprising that the CCR does not predict the properties of formic acid (1) well, so the properties of this compound were not taken into account in the current study. When predicting the physico-chemical and fire hazard properties of organic compounds, intramolecular interatomic interactions are often taken into account $[8,9,11,12]$. The CCR is no exception. These interactions are taken into account by the introduction of a systematic (corrective) amendment [1]. In the case of alkane acids, it was found that the second methyl radical in $\alpha$-position relative to the carboxylic group does not increase the CCC, and in the case of the location of the ethyl, propyl or butyl substituent in $\alpha$-position the increase of the CCC is adjusted minus 0.5 .

The isomerization of the alkyl radical for carboxylic acids, as well as for other classes of organic compounds, which have been previously explored [1,5-7], does not significantly affect the change in the flammability limits and the heat of combustion. That's why, the equations (5)-(7) depending on the total number of carbon atoms in the molecule $\left(N_{c}\right)$, and not on the CCC, are derived for the calculation of parameters mentioned above.

The equations of comparison (9)-(12) are taken from GOST 12.1.044 [13] for prediction of the flash point, the temperature and the flammability limits. It was shown earlier that the Mendeleev formula (13) for determination of the heat of combustion gives accep Table results of calculation [14], therefore it was used as a method of comparison. ACD/Lab 2014 software package was used to compare the forecasts of the boiling point and the heat of vaporization of carboxylic acids (1)-(77) [15].

\section{The discussion of results}

The results of prediction of physico-chemical and fire hazard properties of carboxylic acids (2)(77) by CCR1, CCR2 and by comparison methods are given in Table 1. The correlation coefficient (r), RMSE (Root Mean Squared Error), AARD (Average Absolute Relative Deviation) [16], average absolute deviation $(\varepsilon)$ and average square deviation $(\sigma)$ are chosen as the comparison criteria [11].

$$
R M S E=\sqrt{\frac{\sum_{i=1}^{n}\left(y_{i}-x_{i}\right)^{2}}{n}} A A R D=\frac{100}{n} \sum_{i=1}^{n} \frac{\left|y_{i}-x_{i}\right|}{x_{i}} \sigma=\sqrt{\frac{1}{n-1} \sum_{i=1}^{n}\left(\left|y_{i}-x_{i}\right|-\varepsilon\right)^{2}} \varepsilon=\frac{1}{n} \sum_{i=1}^{n}\left(\left|y_{i}-x_{i}\right|\right),
$$

where $y_{i}, x_{i}$-calculated and experimental values, $n$ - number of compounds in the sample.

It is seen from the Table 2 that CCR1 and CCR 2 methods give more accurate prediction of physico-chemical and fire hazard properties of carboxylic acids than the normative procedures of GOST 12.1.044-89, Mendeleev and ACD/Lab 2014. The only exception is CCR1 for the calculation of the upper temperature flammability limits.

\section{Conclusion}

The study finds that the proposed CCR1 and CCR2 methods can be used to calculate the physicochemical and fire hazard properties of carboxylic acids. According to the accuracy of the boiling temperature, the flash point, the temperature and the flammability limits, the heats of combustion 
and vaporization of carboxylic acids forecasts, the proposed CCR give better results compared to the methods of comparison (GOST 12.1.044, Mendeleev equation, ACD/Lab 2014). The only exception is CCR1 for the calculation of the upper temperature flammability limits, which shows the comparable results with the standard equation (10c). Unknown physico-chemical and/or fire hazard properties are predicted for a number of carboxylic acids (18)-(77).

\section{References}

1. Алексеев К.С., Алексеев С.Г., Барбин Н.М. Прогнозирования физико-химических и пожароопасных показателей спомощью правил углеродной цепи. 1. Алканали. Журнал Сибирского федерального университета. Химия 2018. Т. 11(2), С. 219-229. [Alexeev K.S., Alexeev S.G., Barbin N.M. Prediction of physical-chemical and fire hazard characteristics bycarbon chain rules. 1. Alkanals. Journal of the Siberian Federal University. Chemistry. 2018. Vol. 11(2), P. 219-229. (In Russ.)]

2. Handbook of Chem informatics. From Data to Knowledge in 4 Volumes. Under Ed. Gasteiger J. Weinheim: Wiley-VCHVerlag GmbH \& Co. KgaA, 2003. 1915 p.

3. Yaws C.L. Thermophysical Properties of Chemicals and Hydrocarbons. Amsterdam: Gulf Professional Publishing, 2014. $991 \mathrm{p}$.

4. DIPPR 801. Available at https://dippr.aiche.org/.

5. Смирнов В.В., Алексеев С.Г., Барбин Н.М. Прогнозирование температуры вспышки диалкиламинов. Журнал Сибирского федерального университета. Химия 2016. Т. 9 (1), С. 68-77. [Smirnov V.V., Alexeev S.G., Barbin N.M. Prediction of the dialkylamine's flash points. Journal of the Siberian Federal University. Chemistry 2016. Vol. 9(1), P. 68-77. (In Russ.)]

6. Алексеев С.Г., Мавлютова Л.К., Кошелев А.Ю., Алексеев К.С., Барбин Н.М. Связь показателей пожарной опасности с химическим строением. ХІІ. Алкилбензолы и диалкилбензолы. Пожаровзрывобезопасность 2014. Т. 23(6), С. 38-46. [Alexeev S.G., Mavlyutova L.K., Koshelev A.Yu., Alexeev K.S., Barbin N.M. Correlation of fire hazard characteristics withchemical structure. XII. Alkyl benzenes and dialkyl benzenes. Pozharovzryvobezopasnost' 2014. Vol. 23(6), P. 38-46. (In Russ.)]

7. Алексеев С.Г., Кошелев А.Ю., Барбин Н.М. Связь показателей пожарной опасности с химическим строением. XVIII. Алкильные производные аминометанола. Пожаровзрывобезопасность 2015. Т. 24(2), C. 36-44. [Alexeev S.G., Koshelev A.Yu., Barbin N.M. Correlation offire hazard characteristics with chemical structure. XVIII. Alkyl derivatives aminoethanol. Pozharovzryvobezopasnost' 2015. Vol. 24(2), P. 36-44. (In Russ.)]

8. Батов Д.В., Мочалова Т.А., Петров А.В. Использование аддитивно-группового метода для расчета температуры вспышки спиртов, кетонов и сложных эфиров. Журнал прикладной химии 2011. T. 84(1), C. 55-60. [Batov D.V., Mochalova T.A., Petrov A.V. Additive group techniques for calculating the flash point of alcohols, ketones and esters. Russian Journal of Applied Chemistry 2011. Vol. 84(1), P. 54-59. (In Russ.)]

9. Батов Д.В., Мочалова Т.А., Петров А.В. Описание и прогнозирование температуры вспышки сложных эфиров в рамкахаддитивно-групповогометода.Пожаровзрывобезопасность 2010. T. 19(2), C. 15-18. [Batov D.V., Mochalova T.A., Petrov A.V. Description and prediction of flash temperature of esters within the limits of additive-group method. Pozharovzryvobezopasnost' 2010. Vol. 19(2), P. 15-18. (In Russ.)] 
10. Общая органическая химия. Под ред. Д. Бартона и У.Д. Оллиса. Москва: Химия, 1983. T. 4.728 c. [Comprehensive organic chemistry. Under Ed. Sir D Barton and and W.D. Ollis. Oxford, Pergamon Press, 1979. Vol. 2. 1344 p. (In Russ.)]

11. Смоляков В.М. Зависимость свойств органических веществ от строения их молекул: расчетно-теоретическое исследование. Дис. ... д-ра хим. наук. Тверь, 1995. 81 с. [Smolyakov V.M. Dependence of the properties of organic substances on the structure of their molecules: a computational and theoretical study. Dr. chem. sci. diss. Tver', 1995, 81 p. (In Russ.). Available at: htps://dlib.rsl. $\mathrm{ru} / 01000024557]$

12. Батов Д.В. Использование аддитивно-группового метода для анализа, систематизации и прогнозирования показателей пожарной опасности горючих жидкостей. Российский химический журнал 2014. T. 58(2), C. 4-14. [Batov D.V. The use of the additive group method for analysis, systematization and prediction of fire hazard indicators of flammable liquids. Rossiyskiy khimicheskiy zhurnal 2014. Vol. 58(2), P. 4-14. (In Russ.)]

13. ГОСТ 12.1.044-89*. ССБТ. Пожаровзрывоопасность веществ и материалов. Номенклатура показателей и методы их определения. М.: Стандартинформ, 2006. 100 с. [State Standard 12.1.044-89*. Occupational Safety Standards System. Fire and Explosion Hazard of Substances and Materials. Moscow: Standartinform, 2006. 100 p. (In Russ.)]

14. Демидов П.Г., Саушев В.С.Горение и свойства горючих веществ. М.: ВИПТШМВДСССР, 1975. 280 c. [Demidov P.G., Saushev V.S. Combustion and properties of combustible substances. Moscow: VIPTShMVD SSSR, 1975. 280 p. (In Russ.)]

15. ACD/Boiling Point. Version for Microsoft Windows. User's guide. Calculating the boiling point, vapor pressure, and related properties. Toronto: Advanced Chemistry Development, 2013. 29 p.

16. GharagheiziaF.,Ilani-KashkouliaP.,AcreeJr.W.E.,MohammadiaA.H.,RamjugernathD.Agroup contribution model for determining the vaporization enthalpy of organic compounds at the standard reference temperature of 298 K. Fluid Phase Equilibria 2013. Vol. 360, P. 279-292. 